\title{
Busemann functions and equilibrium measures in last passage percolation models
}

\author{
Eric Cator • Leandro P. R. Pimentel
}

Received: 10 December 2010 / Revised: 28 March 2011 / Published online: 11 April 2011

(C) The Author(s) 2011. This article is published with open access at Springerlink.com

\begin{abstract}
The interplay between two-dimensional percolation growth models and one-dimensional particle processes has been a fruitful source of interesting mathematical phenomena. In this paper we develop a connection between the construction of Busemann functions in the Hammersley last-passage percolation model with i.i.d. random weights, and the existence, ergodicity and uniqueness of equilibrium (or timeinvariant) measures for the related (multi-class) interacting fluid system. As we shall see, in the classical Hammersley model, where each point has weight one, this approach brings a new and rather geometrical solution of the longest increasing subsequence problem, as well as a central limit theorem for the Busemann function.
\end{abstract}

Keywords Hammersley process - Last passage percolation - Busemann functions · Equilibrium

Mathematics Subject Classification (2000) Primary 60C05 - 60K35;

Secondary 60F05

L. P. R. Pimentel was supported by grant number 613.000.605 from the Netherlands Organisation for Scientific Research (NWO).

E. Cator $(\varangle)$

Delft Institute of Applied Mathematics, Delft University of Technology,

Mekelweg 4, 2628 CD Delft, The Netherlands

e-mail: E.A.Cator@tudelft.nl

L. P. R. Pimentel

Institute of Mathematics, Federal University of Rio de Janeiro,

Caixa Postal 68530, 21941-909 Rio de Janeiro, RJ, Brazil

e-mail: leandro@im.ufrj.br 


\section{Introduction}

In the middle of the fifties Busemann [4] introduced a collection of functions to study geometrical aspects of metric spaces. These functions are induced by a metric $d$, and by a collection of rays (semi-infinite geodesics) as follows: the Busemann function $b_{\varpi}(\cdot)$, with respect to a ray $(\varpi(r))_{r \geq 0}$, is the limit of $d(\varpi(r), \varpi(0))-d(\varpi(r), \cdot)$ as $r$ goes to infinity. Along a ray $\varpi$ the metric $d$ becomes additive. By using the triangle inequality, this implies that the defining sequence is non-decreasing and bounded from above, and so it always converges. Using analogous considerations, one can construct Busemann functions over spaces equipped with a super-additive "metric" $L$ (one needs the reversed triangle inequality). In this work we are particularly interested in geometrical aspects of the following stochastic two-dimensional last passage (super-additive) percolation model: let $\mathbf{P} \subseteq \mathbb{R}^{2}$ be a two-dimensional Poisson random set of intensity one. On each point $\mathbf{p} \in \mathbf{P}$ we put a random positive weight $\omega_{\mathbf{p}}$ and we assume that $\left\{\omega_{\mathbf{p}}: \mathbf{p} \in \mathbf{P}\right\}$ is a collection of i.i.d. random variables, distributed according to a distribution function $F$, which are also independent of $\mathbf{P}$. When $F$ is the Dirac distribution concentrated on 1 (each point has weight 1; we will denote this $F$ by $\left.\delta_{1}\right)$, then we refer to this model as the classical Hammersley model $[1,11]$. For each $\mathbf{p}, \mathbf{q} \in \mathbb{R}^{2}$, with $\mathbf{p}<\mathbf{q}$ (inequality in each coordinate, $\left.\mathbf{p} \neq \mathbf{q}\right)$, let $\Pi(\mathbf{p}, \mathbf{q})$ denote the set of all increasing (or up-right) paths, consisting of points in $\mathbf{P}$, from $\mathbf{p}$ to $\mathbf{q}$, where we exclude all points that share (at least) one coordinate with p. So we consider the points in the rectangle ]p, q], where we leave out the south and the west side of the rectangle. In this probabilistic model, the "metric" (or last-passage time) $L$ between $\mathbf{p}<\mathbf{q}$ is defined by

$$
L(\mathbf{p}, \mathbf{q}):=\max _{\varpi \in \Pi(\mathbf{p}, \mathbf{q})}\left\{\sum_{\mathbf{p}^{\prime} \in \varpi} \omega_{\mathbf{p}^{\prime}}\right\} .
$$

Then $L$ is super-additive,

$$
L(\mathbf{p}, \mathbf{q}) \geq L(\mathbf{p}, \mathbf{z})+L(\mathbf{z}, \mathbf{q}) .
$$

When we consider a path $\varpi$ from $\mathbf{p}$ to $\mathbf{q}$ consisting of increasing points $\left(\mathbf{p}_{1}, \ldots, \mathbf{p}_{n}\right)$, we will view $\varpi$ as the lowest increasing continuous path connecting all the points, starting at $\mathbf{p}$ and ending at $\mathbf{q}$, and then excluding $\mathbf{p}$. This way we can talk about crossings with other paths or with lines. Suppose $\varpi_{1}$ and $\varpi_{2}$ are two different paths between $\mathbf{p}$ and $\mathbf{q}$ that both have the maximal weight $L(\mathbf{p}, \mathbf{q})$. Now suppose that $\left(x_{1}, t_{1}\right) \leq\left(x_{2}, t_{2}\right)$ are two points on the intersection of the two paths, such that the paths do not intersect in the open strip $\left(x_{1}, x_{2}\right) \times \mathbb{R}$. Then one path must lie entirely below the other path in this strip, and the total weight of the two paths in this strip must be equal (otherwise we would be able to construct a "longer" path). This means that we can define the minimum of $\varpi_{1}$ and $\varpi_{2}$ by choosing the lowest path in each such strip. The finite geodesic between $\mathbf{p}$ and $\mathbf{q}$ is given by the lowest path (in the sense we just described) that attains the maximum in the definition of $L(\mathbf{p}, \mathbf{q})$. We will denote this geodesic 
by $\varpi(\mathbf{p}, \mathbf{q})$ (this is well defined for any ordered pair $(\mathbf{p}, \mathbf{q})$, even if we do not specify the order).

Given this function $L$, we can define an interacting fluid process in the following way (for a precise description), see Sect. 4: consider the set $\mathcal{N}$ of all increasing right-continuous functions $v$ on $\mathbb{R}$ such that $v(0)=0$ and

$$
\liminf _{x \rightarrow-\infty} v(x) / x>0
$$

To every $v \in \mathcal{N}$ we can associate a measure, which we also denote by $v$, such that $v((x, y])=v(y)-v(x)$. We will consider $v \in \mathcal{N}$ as a starting configuration for our interacting fluid process at time $t=0$; the configuration at time $t$ is denoted by $M_{t}^{v}$ (so $M_{0}^{v}=v$ ). The evolution of the process can be described as follows: suppose there is a Poisson point at $(x, t)$, for some $t>0$, with weight $w$. Denote $M_{t-}^{v} \in \mathcal{N}$ as the configuration just before time $t$. Then

$$
M_{t}^{v}(y)= \begin{cases}M_{t-}^{v}(y) & \text { if } y<x \\ \max \left(M_{t-}^{v}(y), M_{t-}^{v}(x)+w\right) & \text { if } y \geq x\end{cases}
$$

This means that if at time $t$ and at position $x$ a Poisson point appears with weight $w$, then at time $t$, we add mass $w$ at position $x$ of our configuration, and we take this mass away from the positions closest to the right of $x$; see Fig. 1 in Sect. 4 for an example where $v$ has only atoms. When $F=\delta_{1}$ (the classical Hammersley process), we simply retrieve the classical Hammersley interacting particle process. We will show the following connection between the interacting fluid process $M_{t}^{v}$ and the function $L$ (Proposition 4.1): define for $x \in \mathbb{R}$ and $t \geq 0$

$$
L_{v}(x, t)=\sup _{z \leq x}\{v(z)+L((z, 0),(x, t))\}
$$

Then

$$
M_{t}^{v}((x, y])=L_{v}(y, t)-L_{v}(x, t) .
$$

In Corollary 4.4 and Theorem 5.3 we construct a one parameter family of processes $v_{\alpha} \in \mathcal{N}$ that are the unique ergodic equilibrium measures for the Hammersley interacting fluid process. This construction uses a concept known as Busemann functions, which we will describe in the next paragraph. We view this connection between the Busemann functions and the interacting fluid process as the key idea of this paper. On the one hand, it allows us to prove existence, uniqueness and ergodicity (mixing) of the equilibrium measures of the interacting fluid process. Furthermore, it gives us a natural way to prove a strong law of large numbers for the second class particle and to define a multi-class fluid system with a countable number of classes, which is a new result even in the classical Hammersley process. On the other hand, it implies a central limit theorem for the Busemann function in the classical model. This result also shows a phase transition from a Gaussian limit distribution, on the square-root scale, to Tracy-Widom type (zero-mean) limit distribution, on the cube-root scale, 
at the critical angle $\alpha-\pi$; here we use the results from [2]. This transition from square-root to cube-root scaling for Busemann functions was first conjectured in 2001 by Howard and Newman [12] (see also [16]). Furthermore, we conjecture that under suitable conditions, the random measures $v_{\alpha}$ behave asymptotically like a Brownian motion plus linear drift (just like a compound Poisson process). If this is true, we believe that we can extend the methods from [6] to conclude cube-root asymptotics for the length of the longest paths and its fluctuation. This idea will be pursued in an upcoming paper.

Throughout this paper, except in Theorem 2.1, we will make the following assumption on the distribution function $F$ of the weights:

$$
\int_{0}^{\infty} \exp (a x) d F(x)<+\infty, \text { for some } a>0 \text {. }
$$

We will work from this assumption in all statements. In [7], using methods developed by Newman and co-authors in [12] and [14] also applied to the classical Hammersley process by Wüthrich in [16], and using ideas from Kesten in [13], it is shown that finite geodesics can be extended to semi-infinite $\alpha$-rays by moving one endpoint appropriately to infinity. An $\alpha$-ray starting at $\mathbf{p} \in \mathbb{R}^{2}$, denoted by $\varpi_{\alpha}(\mathbf{p})$, is a semi-infinite geodesic that starts at $\mathbf{p}$ and moves to infinity in the direction $\vec{\alpha}:=(\cos \alpha, \sin \alpha)$, for $\alpha \in(\pi, 3 \pi / 2)$. It turns out that under Assumption (1.1), for fixed $\alpha$, with probability 1 , each $\mathbf{p} \in \mathbb{R}^{2}$ is the starting point of a unique $\alpha$-ray, and two $\alpha$-rays will always coalesce eventually. In Sect. 2 we will state precisely the theorems for existence and coalescence of $\alpha$-rays. An important tool in the development of these concepts is the following result, known as the shape theorem: there exists a constant $\gamma=\gamma(F)$ such that for all $x, t \geq 0$

$$
\lim _{r \rightarrow \infty} \frac{L(\mathbf{0}, r(x, t))}{r}=\gamma \sqrt{x t}(\text { a.s. })
$$

In Sect. 3 we will use the $\alpha$-rays to construct the Busemann function $B_{\alpha}:$ if $\mathbf{x}, \mathbf{y} \in \mathbb{R}^{2}$, let $\mathbf{c}=\mathbf{c}(\alpha, \mathbf{x}, \mathbf{y})$ be the coalescence point of the two $\alpha$-rays starting at $\mathbf{x}$ and $\mathbf{y}$. Then

$$
B_{\alpha}(\mathbf{x}, \mathbf{y})=L(\mathbf{c}, \mathbf{y})-L(\mathbf{c}, \mathbf{x}) .
$$

It is not hard to see that the distribution of $B_{\alpha}$ is invariant under translation and that $B_{\alpha}$ is additive: $B_{\alpha}(\mathbf{x}, \mathbf{z})=B_{\alpha}(\mathbf{x}, \mathbf{y})+B_{\alpha}(\mathbf{y}, \mathbf{z})$. Its most important property, however, is the following connection with the interacting fluid process: if we define the measures $v_{\alpha}^{t}$ on $\mathbb{R}$ by

$$
v_{\alpha}^{t}((x, y])=B_{\alpha}((x, t),(y, t))
$$

then the family of random measures $\left\{v_{\alpha}^{t}: t \in \mathbb{R}\right\}$ forms a Markov process, and in fact we have that if we define $v_{\alpha}=v_{\alpha}^{0}$, then $M_{t}^{v_{\alpha}}=v_{\alpha}^{t}$ (Corollary 4.4). The translation invariance of the Busemann function then shows that $v_{\alpha}$ is an ergodic equilibrium measure for the interacting fluid process. 
In Sect. 8 we describe how to define a Busemann function in the last passage percolation on the lattice $\mathbb{Z}^{2}$ with i.i.d. weights on the lattice points. It is an important open question for a long time already, how to prove in this general set-up that the shape function, see (1.2), is strictly curved, a fact we need to define our $\alpha$-rays. However, if we restrict ourselves to exponential (or geometric) weights, in which case the last passage percolation is an alternative description of the totally asymmetric exclusion process (TASEP), we know the shape function, and it is indeed strictly curved. Therefore, we can define the Busemann function and we find a similar connection to the (known) equilibrium measures, which allows us to prove analogous results.

We feel that the connection between the Busemann functions and the equilibrium measures gives us an important new tool to study last passage percolation and the corresponding fluid processes. In an upcoming paper, we will use the Busemann function and the results from this paper to determine the asymptotic speed of a second class particle, given a deterministic, rarefaction initial condition, in the classical Hammersley process and in TASEP. Furthermore, as said before, we have strong indications that the Busemann function can help us establish the cube-root behavior of the length of a longest path, and the fluctuations of the longest path.

Overview. In Sect. 2 we will state the theorems, that we will use further on, about the limit shape and the existence and coalescence of $\alpha$-rays, and then give precise references for the proofs. In Sect. 3 we define the Busemann function and give its most important properties. In Sect. 4 we introduce the Hammersley interacting fluid process and establish the connection between the Busemann function and the equilibrium measures. In Sect. 5 we prove uniqueness and ergodicity (mixing property) of the equilibrium measures, and we show local convergence to the equilibrium measure in case of a rarefaction fan. In Sect. 6 we prove the central limit theorem for the Busemann function in the classical model. In Sect. 7, we show how we can define a multi-class system with a countable number of classes, and we establish the strong law for a second class particle. In Sect. 8 we state the analogous results for the TASEP.

\section{Shape function and $\alpha$-rays}

A key notion in this paper will be an $\alpha-$ ray: for each angle $\alpha \in(\pi, 3 \pi / 2)$ and for each point $\mathbf{x} \in \mathbb{R}^{2}, \varpi_{\alpha}(\mathbf{x})$ is the lowest continuous down-left path through an ordered sequence $\left(\mathbf{p}_{i}\right)_{i \geq 0}$ in $\mathbb{R}^{2}$, with $\mathbf{p}_{0}=\mathbf{x}, \mathbf{p}_{i} \in \mathbf{P}$ and $\mathbf{p}_{i} \geq \mathbf{p}_{j}$ whenever $i \leq j$. Furthermore, $\varpi\left(\mathbf{p}_{j}, \mathbf{p}_{i}\right) \subset \varpi_{\alpha}(\mathbf{x})$ (every part of the path is a geodesic), and finally we must have that

$$
\lim _{i \rightarrow \infty} \frac{\mathbf{p}_{i}}{\left\|\mathbf{p}_{i}\right\|}=\vec{\alpha}:=(\cos \alpha, \sin \alpha)
$$

A crucial step for the existence of $\alpha$-rays is the following shape theorem: set $\mathbf{0}=$ $(0,0), \mathbf{n}=(n, n)$,

$$
F(x)=\mathbb{P}\left(\omega_{\mathbf{p}} \leq x\right) \text { and } \gamma=\gamma(F)=\sup _{n \geq 1} \frac{\mathbb{E}(L(\mathbf{0}, \mathbf{n}))}{n}>0 .
$$


Theorem 2.1 Suppose that

$$
\int_{0}^{\infty} \sqrt{1-F(x)} d x<+\infty
$$

Then $\gamma(F)<\infty$ and for all $x, t>0$, as $r \rightarrow \infty$,

$$
\frac{L(\mathbf{0},(r x, r t))}{r} \rightarrow \gamma \sqrt{x t} \text { a.s. and } \frac{\mathbb{E} L(\mathbf{0},(r x, r t))}{r} \rightarrow \gamma \sqrt{x t}
$$

Further, if (2.2) is strengthened to: there exists $a>0$ such that

$$
\int_{0}^{\infty} \exp (a x) d F(x)<+\infty
$$

then there exist constants $c_{0}, c_{1}, c_{2}, c_{3}, c_{4}>0$ such that for all $r \geq c_{0}$

$$
\mathbb{P}(|L(\mathbf{0},(r, r))-\gamma r| \geq u) \leq c_{1} \exp \left(-c_{2} \frac{u}{\sqrt{r} \log r}\right)
$$

for $u \in\left[c_{3} \sqrt{r} \log ^{2} r, c_{4} r^{3 / 2} \log r\right]$.

For proofs see Theorems 1.1 and 1.2 in [7]. Theorem 2.1 shows that $L$ has a curved limiting shape, mainly due to the invariance of the Poisson process under volume preserving maps: if $x, t, r>0$ and $\mathbf{p} \in \mathbb{R}^{2}$, then

$$
L(\mathbf{0},(x, t)) \stackrel{\mathcal{D}}{=} L(\mathbf{p}, \mathbf{p}+(r x, t / r))
$$

This is because under this map, the distribution of the Poisson process does not change, and the up-right paths are preserved. The almost sure convergence is a standard consequence of the sub-additive ergodic theorem, once we have a bound on $\mathbb{E}(L(\mathbf{0}, \mathbf{n}))$, linear in $n$.

The following theorem gives us existence and coalescence of $\alpha$-rays. Before we state the theorem, we shall define what we mean by convergence of paths: we say that a sequence of paths $\varpi^{n}$ converges to $\varpi$, and denote $\lim _{n \rightarrow \infty} \varpi^{n}=\varpi$, if for all bounded subsets $B \subset \mathbb{R}^{2}$ there exists $n_{0}$ such that $\varpi^{n} \cap B=\varpi \cap B$ for all $n \geq n_{0}$.

Theorem 2.2 Assume (1.1), so for some a $>0$,

$$
\int_{0}^{\infty} \exp (a x) d F(x)<+\infty
$$

Then for fixed $\alpha \in(\pi, 3 \pi / 2)$ the following holds with probability one: 
(1) For each $\mathbf{x} \in \mathbb{R}^{2}$ there exists a unique $\alpha$-ray starting from $\mathbf{x}$, which we denote by $\varpi_{\alpha}(\mathbf{x})$.

(2) For any sequence $\left(\mathbf{z}_{n}\right)_{n \geq 0}$ of points in $\mathbb{R}^{2}$ with $\left\|\mathbf{z}_{n}\right\| \rightarrow \infty$,

$$
\text { if } \lim _{n \rightarrow \infty} \frac{\mathbf{z}_{n}}{\left\|\mathbf{z}_{n}\right\|}=(\cos \alpha, \sin \alpha) \text { then } \lim _{n \rightarrow \infty} \varpi\left(\mathbf{x}, \mathbf{z}_{n}\right)=\varpi_{\alpha}(\mathbf{x}) \text {. }
$$

(3) For all $\mathbf{x}, \mathbf{y} \in \mathbb{R}^{2}$ there exists $\mathbf{c}=\mathbf{c}(\alpha, \mathbf{x}, \mathbf{y})$ such that $\varpi_{\alpha}(\mathbf{x})$ and $\varpi_{\alpha}(\mathbf{y})$ coalesce at $\mathbf{c}$ :

$$
\varpi_{\alpha}(\mathbf{x})=\varpi(\mathbf{x}, \mathbf{c}) \cup \varpi_{\alpha}(\mathbf{c}) \text { and } \varpi_{\alpha}(\mathbf{y})=\varpi(\mathbf{y}, \mathbf{c}) \cup \varpi_{\alpha}(\mathbf{c}) \text {. }
$$

The proof of Theorem 2.2 is based on a method introduced by Newman [14] that can be applied in a wide percolation context. An outline of the proof is given in [7]. A detailed proof of the same theorem, but now restricted to the Hammersley classical model, can be found in [16]. Finally, note that two $\alpha$-rays can only coalesce once: if they have two points $\mathbf{p} \geq \mathbf{q}$ in common, then they both must coincide with $\varpi(\mathbf{q}, \mathbf{p})$ in between, by definition of the $\alpha$-ray.

\section{The Busemann function}

Using the concept of $\alpha$-rays, we will study the function $B_{\alpha}(\mathbf{x}, \mathbf{y})$, which is defined by taking the first coalescence point $\mathbf{c}=\mathbf{c}(\alpha, \mathbf{x}, \mathbf{y})$ between the $\alpha$-ray that starts from $\mathbf{x}$ and the one that starts from $\mathbf{y}$ (remember that these two rays coalesce), and setting

$$
B_{\alpha}(\mathbf{x}, \mathbf{y})=L(\mathbf{c}, \mathbf{y})-L(\mathbf{c}, \mathbf{x}) .
$$

Note that if we take a different coalescence point $\mathbf{c}^{\prime}$, then $\mathbf{c} \geq \mathbf{c}^{\prime}$ and they both lie on a geodesic. Since $L$ is additive on a geodesic, we get $L\left(\mathbf{c}^{\prime}, \mathbf{x}\right)=L\left(\mathbf{c}^{\prime}, \mathbf{c}\right)+L(\mathbf{c}, \mathbf{x})$, which shows that the definition of $B_{\alpha}(\mathbf{x}, \mathbf{y})$ does not depend on the choice of the coalescence point. Let $\left(\mathbf{z}_{n}\right)_{n \geq 1}$ be any unbounded decreasing sequence that follows direction $(\cos \alpha, \sin \alpha)$, and let $\mathbf{c}\left(\mathbf{z}_{n}, \mathbf{x}, \mathbf{y}\right)$ denote the most up-right coalescence point between $\varpi\left(\mathbf{z}_{n}, \mathbf{x}\right)$ and $\varpi\left(\mathbf{z}_{n}, \mathbf{y}\right)$. By Theorem 2.2, with probability one, there exists $n_{0}>0$ such that

$$
\forall n \geq n_{0} \quad \mathbf{c}\left(\mathbf{z}_{n}, \mathbf{x}, \mathbf{y}\right)=\mathbf{c}(\alpha, \mathbf{x}, \mathbf{y}) \text { and } L\left(\mathbf{z}_{n}, \mathbf{y}\right)-L\left(\mathbf{z}_{n}, \mathbf{x}\right)=B_{\alpha}(\mathbf{x}, \mathbf{y})
$$

Therefore, in geometrical terms, $B_{\alpha}(\mathbf{x}, \cdot)$ can be seen as the Busemann function along the ray $\varpi_{\alpha}(\mathbf{x})$.

Some properties of $B_{\alpha}$ are summarized in the following proposition. The proofs can be found in Sect. 9.

Proposition 3.1 Define the Busemann function $B_{\alpha}$ as above, for $\alpha \in(\pi, 3 \pi / 2)$.

(1) The distribution of the function $B_{\alpha}$ is translation invariant: $\forall \mathbf{p} \in \mathbb{R}^{2}$

$$
B_{\alpha}(\cdot+\mathbf{p}, \cdot+\mathbf{p}) \stackrel{\mathcal{D}}{=} B_{\alpha}(\cdot, \cdot)
$$


(2) $B_{\alpha}$ is anti-symmetric and additive: $\forall \mathbf{x}, \mathbf{y}, \mathbf{z} \in \mathbb{R}^{2}$

$$
B_{\alpha}(\mathbf{x}, \mathbf{y})=-B_{\alpha}(\mathbf{y}, \mathbf{x}) \text { and } B_{\alpha}(\mathbf{x}, \mathbf{z})=B_{\alpha}(\mathbf{x}, \mathbf{y})+B_{\alpha}(\mathbf{y}, \mathbf{z})
$$

(3) For any $(x, t) \in \mathbb{R}^{2}$,

$$
B_{5 \pi / 2-\alpha}(\mathbf{0},(x, t)) \stackrel{\mathcal{D}}{=} B_{\alpha}(\mathbf{0},(t, x)) .
$$

(4) For any $(x, t) \in \mathbb{R}^{2}$, all $\alpha \in(\pi, 3 \pi / 2)$ and $\rho>0$, we have

$$
B_{\alpha}((0,0),(x, t)) \stackrel{\mathcal{D}}{=} B_{\tilde{\alpha}}\left((0,0),\left(\rho x, \rho^{-1} t\right)\right),
$$

where $\tan \tilde{\alpha}=\rho^{-2} \tan \alpha$.

(5) If $\mathbf{x} \leq \mathbf{y}$ and $\mathbf{x} \neq \mathbf{y}$, then

$$
B_{\alpha}(\mathbf{x}, \mathbf{y}) \geq 0 \text { and } 0<\mathbb{E}\left(B_{\alpha}(\mathbf{x}, \mathbf{y})\right)<+\infty
$$

(6) Fix $\mathbf{x}, \mathbf{y} \in \mathbb{R}^{2}$ and $\mathbf{p}, \mathbf{q} \in \mathbb{R}^{2}$ such that $\mathbf{p}, \mathbf{q} \geq \mathbf{0}$. The function $\lambda \mapsto B_{\alpha}(\mathbf{x}+$ $\lambda \mathbf{p}, \mathbf{y}+\lambda \mathbf{q}$ ) is càdlàg in $\lambda \in \mathbb{R}$.

As mentioned in Sect. 1, the most important aspect of $B_{\alpha}$ is a Markovian structure described in the following proposition.

Proposition 3.2 For all $s \leq t$ and $x \in \mathbb{R}$ we have

$$
B_{\alpha}((0, s),(x, t))=\sup _{z \leq x}\left\{B_{\alpha}((0, s),(z, s))+L((z, s),(x, t))\right\}
$$

Proof Without loss of generality we can take $s=0$ (and therefore $t \geq 0$ ). Define $Z_{\alpha}=Z_{\alpha}(x, t) \in \mathbb{R}$ as the crossing-point of the $\alpha$-ray starting at $(x, t)$ with the $x$-axis. Clearly, $Z_{\alpha} \leq x$ and

$$
\begin{aligned}
B_{\alpha}(\mathbf{0},(x, t)) & =B_{\alpha}\left(\mathbf{0},\left(Z_{\alpha}, 0\right)\right)+B_{\alpha}\left(\left(Z_{\alpha}, 0\right),(x, t)\right) \\
& =B_{\alpha}\left(\mathbf{0},\left(Z_{\alpha}, 0\right)\right)+L\left(\left(Z_{\alpha}, 0\right),(x, t)\right) .
\end{aligned}
$$

The last equality follows from the fact that $(x, t)$ and $\left(Z_{\alpha}, 0\right)$ are lying on an $\alpha$-ray. This means that it is enough to prove that for all $z \leq x$,

$$
B_{\alpha}(\mathbf{0},(z, 0))+L((z, 0),(x, t)) \leq B_{\alpha}\left(\mathbf{0},\left(Z_{\alpha}, 0\right)\right)+L\left(\left(Z_{\alpha}, 0\right),(x, t)\right) .
$$

Suppose $\mathbf{p}$ is a coalescence point of the $\alpha$-rays starting at $\mathbf{0},(x, t)$ and $(z, 0)$. Then

$$
\begin{aligned}
B_{\alpha}(\mathbf{0},(z, 0)) & =L(\mathbf{p},(z, 0))-L(\mathbf{p}, \mathbf{0}) \text { and } \\
B_{\alpha}\left(\mathbf{0},\left(Z_{\alpha}, 0\right)\right) & =L\left(\mathbf{p},\left(Z_{\alpha}, 0\right)\right)-L(\mathbf{p}, \mathbf{0}) .
\end{aligned}
$$


Furthermore, since $\mathbf{p},\left(Z_{\alpha}, 0\right)$ and $(x, t)$ are elements of $\varpi_{\alpha}(x, t)$, we know that

$$
\begin{aligned}
L(\mathbf{p},(z, 0))+L((z, 0),(x, t)) & \leq L(\mathbf{p},(x, t)) \\
& =L\left(\mathbf{p},\left(Z_{\alpha}, 0\right)\right)+L\left(\left(Z_{\alpha}, 0\right),(x, t)\right) .
\end{aligned}
$$

From this, (3.3) easily follows.

Define the positive measures $v_{t}^{\alpha}$ on $\mathbb{R}$, using Proposition 3.1, by

$$
\begin{aligned}
v_{t}^{\alpha}((x, y]) & :=B_{\alpha}((x, t),(0,0))-B_{\alpha}((y, t),(0,0)) \\
& =B_{\alpha}((x, t),(y, t)) \quad \forall x \leq y .
\end{aligned}
$$

Proposition 3.2 then shows that the process $t \mapsto v_{t}^{\alpha}$ is a Markov process: the future evolution of $v_{t}^{\alpha}$ depends on the Poisson process in the upper-half plane $\mathbb{R} \times(t, \infty)$ and on the present value of $v_{t}^{\alpha}$, not on the past of the process (which is of course independent of the Poisson process in $\mathbb{R} \times(t, \infty))$. Note that the distribution of $v_{t}^{\alpha}$ does not depend on $t$ (by Proposition 3.1(1)), so this distribution is an equilibrium (or time invariant) measure for the underlying Markov process. In the next section we will describe the generator of this Markov process, which will be an extension of the classical Hammersley interacting particle process.

\section{The Hammersley interacting fluid system}

It is well known that the classical Hammersley model, where all weights are 1, described in Aldous and Diaconis [1], has a representation as an interacting particle system. The Hammersley process with random weights has a similar description, although a better name might be an interacting fluid system. We start by restricting the compound Poisson process $\left\{\omega_{\mathbf{p}}: \mathbf{p} \in \mathbf{P}\right\}$ to $\mathbb{R} \times \mathbb{R}_{+}$. Then we choose a positive, locally finite measure $v$ defined on $\mathbb{R}$. Usually, these measures will be purely atomic, but this is not necessary. To each measure $v$ we associate a non-decreasing process $v(\cdot)$ defined by

$$
v(x)= \begin{cases}v((0, x]) & \text { for } x \geq 0 \\ -v((x, 0]) & \text { for } x<0\end{cases}
$$

Note that $v(\cdot)$ is a cadlag function. Although the details are a bit cumbersome, all the results we will show can be extended, mutatis mutandis, to the case where $v(x)=-\infty$ for $x<0$, which would correspond to a non-locally finite measure with an infinite fluid density to the left of 0 . This is a quite natural starting condition, but we will not use it explicitly in this paper.

The Hammersley interacting fluid system $\left(M_{t}^{\nu}: t \geq 0\right)$ is a stochastic process with values in the space of positive, locally finite measures on $\mathbb{R}$. Its evolution is defined as follows: if there is a Poisson point with weight $\omega$ at a point $\left(x_{0}, t\right)$, then 


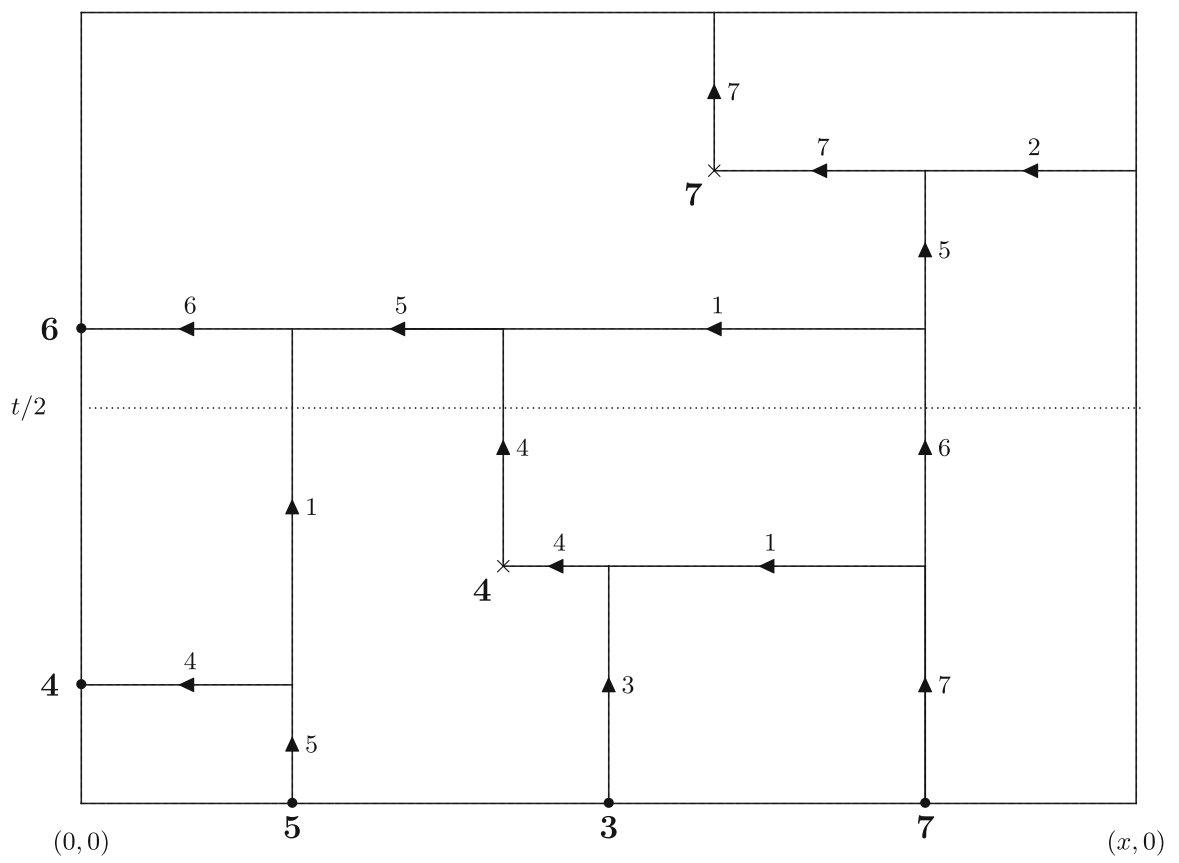

Fig. 1 Example of the Hammersley interacting fluid process

$M_{t}^{\nu}\left(\left\{x_{0}\right\}\right)=M_{t-}^{\nu}\left(\left\{x_{0}\right\}\right)+\omega$, and for $x>x_{0}$,

$$
M_{t}^{v}\left(\left(x_{0}, x\right]\right)=\left(M_{t-}^{v}\left(\left(x_{0}, x\right]\right)-\omega\right)_{+} .
$$

Here, $M_{t-}^{v}$ is the "mass distribution" of the fluid at time $t$ if the Poisson point at $\left(x_{0}, t\right)$ would be removed. To the left of $x_{0}$ the measure does not change. In words, the Poisson point at $\left(x_{0}, t\right)$ moves a total mass $\omega$ to the left, to the point $x_{0}$, taking the mass from the first available fluid to the right of $x_{0}$. See Fig. 1 for a visualization, in case of atomic measures, of the process inside a space-time box. In this picture, restricted to $[0, x]$, the measure $v$ consists of three atoms of weight 5, 3 and 7. The measure $M_{t / 2}^{v}$ consists of three atoms of weight 1, 4 and 6, while at time $t$, it consists of one atom with weight 7.

It is not true that the evolution $M_{t}$ is well defined for all measures $v$ (e.g. if we start with a finite number of particles to the left of 0 , every particle would be pulled instantaneously to $-\infty$ ). In this paper we follow the Aldous and Diaconis [1] graphical representation in the last-passage model (compare to the result in the classical case, found in their paper):

Proposition 4.1 Let $\mathcal{N}$ be the set of all positive, locally finite measures $v$ such that

$$
\liminf _{y \rightarrow-\infty} \frac{v(y)}{y}>0 .
$$


For each $v \in \mathcal{N}$, the process defined by

$$
L_{v}(x, t):=\sup _{z \leq x}\{v(z)+L((z, 0),(x, t))\} \quad(x \in \mathbb{R}, t \geq 0)
$$

is well defined and the measure

$$
M_{t}^{v}((x, y]):=L_{v}(y, t)-L_{v}(x, t)
$$

evolves according to the Hammersley interacting fluid system.

Proof It follows immediately from the definition that $L_{v}(x, t)$ is increasing in $x$ and $t$, even if $L_{v}$ would not be finite everywhere. This implies that if we can prove that $L_{v}$ is finite on for example $\mathbb{Z} \times \mathbb{Z}_{+}$with probability one, then almost surely, $L_{v}$ is finite everywhere. Therefore, we need only prove for any fixed point $(x, t)$ that $L_{v}(x, t)$ is finite with probability one. Use (2.4) to see that for any $\varepsilon>0$ and $z \leq x$,

$$
\mathbb{P}(L((z, 0),(x, t))>-\varepsilon z)=\mathbb{P}(L((0,0),(\sqrt{(x-z) t}, \sqrt{(x-z) t}))>-\varepsilon z) .
$$

Defining $r=\sqrt{(x-z) t}$ and using Theorem 2.1, we get

$$
\mathbb{P}(L((z, 0),(x, t))>-\varepsilon z)=\mathbb{P}\left(L((0,0),(r, r))>\varepsilon\left(-x+r^{2} / t\right)\right) \leq c_{1} e^{-c_{2} r},
$$

for some positive constants $c_{1}$ and $c_{2}$, and for $r$ big enough. Borel-Cantelli then shows that

$$
\frac{L((z, 0),(x, t))}{-z} \stackrel{\text { a.s. }}{\longrightarrow} 0 \quad(z \rightarrow-\infty) .
$$

Now (4.3) gives us the desired result.

We can show that $x \mapsto L_{v}((z, 0),(x, t))$ is càdlàg using the fact that for $y \geq x$,

$$
\begin{aligned}
& L((z, 0),(y, t)) \leq L((z, 0),(x, t))+L((x+, 0),(y, t)) \text { and } \\
& \quad \lim _{y \downarrow x} L((x+, 0),(y, t))=0,
\end{aligned}
$$

where $x+$ means that you are not allowed to use a possible Poisson point directly above $x$. Therefore, $M_{t}^{v}$ is indeed a locally finite measure on $\mathbb{R}$. To see that $M_{t}^{v}$ follows the Hammersley interacting fluid dynamics that we have just defined, suppose that there is a Poisson point in $\left(x_{0}, t\right)$ with weight $\omega$. For $x<x_{0}$, this Poisson point has no effect, so $M_{t}^{v}=M_{t-}^{v}$ on $\left(-\infty, x_{0}\right)$. Clearly,

$$
M_{t}^{v}\left(\left\{x_{0}\right\}\right)=M_{t-}^{v}\left(\left\{x_{0}\right\}\right)+\omega .
$$

If $x>x_{0}$, then the longest path to $(x, t)$ that attains the supremum in (4.4) can either use the weight in $\left(x_{0}, t\right)$, which would give $L_{v}(x, t)=L_{v}\left(x_{0}, t\right)=L_{v}\left(x_{0}, t-\right)+\omega$, 
or it could ignore the weight in $\left(x_{0}, t\right)$, which would give $L_{v}(x, t)=L_{v}(x, t-)$. This proves that

$$
\begin{aligned}
M_{t}^{v}\left(\left(x_{0}, x\right]\right) & =L_{v}(x, t)-L_{v}\left(x_{0}, t\right) \\
& =\max \left\{L_{v}\left(x_{0}, t-\right)+\omega, L_{v}(x, t-)\right\}-L_{v}\left(x_{0}, t-\right)-\omega \\
& =\left(M_{t-}^{v}\left(\left(x_{0}, x\right]\right)-\omega\right)_{+} .
\end{aligned}
$$

We have two remarks concerning the interactive fluid process. The first remark concerns the flux of the fluid system through the $t$-axis. We define the flux measure $v^{*}$ on $[0, \infty)$ such that for $t \geq s \geq 0$,

$$
v^{*}((s, t])=L_{v}(0, t)-L_{v}(0, s) .
$$

In Fig. 1 we can see that $v^{*}((0, t])=10$, whereas $v^{*}((0, t / 2])=4$. We can compare $v^{*}$ to the process of sinks in the classical Hammersley process with sources and sinks, as defined in [5]. We then have the following lemma.

Lemma 4.2 For $x, t \geq 0$,

$$
L_{v}(x, t)=\max \left\{\sup _{0 \leq z \leq x}\{v(z)+L((z, 0),(x, t))\}, \sup _{0 \leq z \leq t}\left\{v^{*}(z)+L((0, z),(x, t))\right\}\right\} .
$$

The way to prove Lemma 4.2 leads us to our second remark: the supremum in the definition of $L_{v}(x, t)$ is actually attained (it is therefore a maximum). This is the statement of the following lemma.

Lemma 4.3 With probability 1 , the set $\left\{z \leq x: L_{v}(x, t)=v(z)+L((z, 0),(x, t))\right\}$ is non-empty, for all $(x, t) \in \mathbb{R} \times[0, \infty)$. Furthermore, if we define

$$
Z_{v}(x, t)=\sup \left\{z \leq x: L_{v}(x, t)=v(z)+L((z, 0),(x, t))\right\},
$$

then

$$
L_{v}(x, t)=v\left(Z_{v}(x, t)\right)+L\left(\left(Z_{v}(x, t), 0\right),(x, t)\right) .
$$

Proof This lemma relies on the fact that on a compact interval, the sum of a nondecreasing right-continuous function $(v(z))$ and a non-increasing left-continuous function $(z \mapsto L((z, 0),(x, t)))$ attains its maximum, and the set of maxima is closed (see Lemma 9.1 in Sect. 9). Now we have to show that with probability 1, for all $(y, s) \in \mathbb{R} \times[0, \infty)$, the supremum over $z \leq y$ can actually be restricted to a compact set. For a single $(x, t)$ (and therefore for a countable set of $(x, t)$ 's), this has been 
done in the proof of Proposition 4.1, using (4.3) and Theorem 2.1 for $L$. Then we can conclude the desired compactness property for all $(y, s)$ by using the inequality

$$
\begin{aligned}
& v(z)+L((z, 0),(y, s)) \leq v\left(Z_{v}(x, t)\right)+L\left(\left(Z_{v}(x, t), 0\right),(y, s)\right) \\
& \forall z \leq Z_{v}(x, t), y \geq x, s \leq t .
\end{aligned}
$$

This last inequality can be seen as follows: suppose there exists $z<Z_{v}(x, t)$ such that

$$
v(z)+L((z, 0),(y, s))>v\left(Z_{v}(x, t)\right)+L\left(\left(Z_{v}(x, t), 0\right),(y, s)\right) .
$$

Then the finite geodesic $\varpi((z, 0),(y, s))$ must intersect the finite geodesic $\varpi\left(\left(Z_{v}(x, t), 0\right),(x, t)\right)$ in some point $\mathbf{c}$. It follows that $L((z, 0),(y, s))=L((z, 0), \mathbf{c})$ $+L(\mathbf{c},(y, s))$, and $L\left(\left(Z_{v}(x, t), 0\right),(x, t)\right)=L\left(\left(Z_{v}(x, t), 0\right), \mathbf{c}\right)+L(\mathbf{c},(x, t))$. Furthermore, we have that

$$
v(z)+L((z, 0),(y, s))>v\left(Z_{v}(x, t)\right)+L\left(\left(Z_{v}(x, t), 0\right), \mathbf{c}\right)+L(\mathbf{c},(y, s)) .
$$

Combining this gives

$$
\begin{aligned}
v(z)+L((z, 0),(x, t)) & \geq v(z)+L((z, 0), \mathbf{c})+L(\mathbf{c},(x, t)) \\
& >v\left(Z_{v}(x, t)\right)+L\left(\left(Z_{v}(x, t), 0\right), \mathbf{c}\right)+L(\mathbf{c},(x, t)) \\
& =v\left(Z_{v}(x, t)\right)+L\left(\left(Z_{v}(x, t), 0\right),(x, t)\right),
\end{aligned}
$$

which contradicts the definition of $Z_{v}(x, t)$.

Note that we have also proved the following statement:

$$
\forall y \geq x \forall 0 \leq s \leq t: Z_{v}(y, s) \geq Z_{v}(x, t) .
$$

Proof of Lemma 4.2: Define $v_{-}$as the restriction of $v$ to $(-\infty, 0]$ (so $v_{-}$has no mass on the positive $x$-axis). Then for $x, t \geq 0$, there exists $Z \leq 0$, so that

$$
L_{\nu_{-}}(x, t)=v(Z)+L((Z, 0),(x, t)) .
$$

The finite geodesic $\varpi((Z, 0),(x, t))$ crosses the positive $t$-axis in some point, call it $(0, s)$. It is not hard to see that $v^{*}(s)=v(Z)+L((Z, 0),(0, s))$ and

$$
L_{\nu_{-}}(x, t)=v^{*}(s)+L((0, s),(x, t)) \geq v^{*}(\tilde{s})+L((0, \tilde{s}),(x, t)) \quad(\forall 0 \leq \tilde{s} \leq t) .
$$

This follows from similar arguments as at the end of the proof of Lemma 4.3. Finally, we remark that

$$
L_{v}(x, t)=\max \left\{\sup _{z \leq 0}\left\{v_{-}(z)+L((z, 0),(x, t))\right\}, \sup _{z>0}\{v(z)+L((z, 0),(x, t))\}\right\},
$$

from which Equation (4.7) follows. 
Assume that we have a probability measure defined on $\mathcal{N}$ and consider $v \in \mathcal{N}$ as a realization of this probability measure. We say that $v$ is time invariant for the Hammersley interacting fluid process (in law) if

$$
M_{t}^{v} \stackrel{\mathcal{D}}{=} M_{0}^{v}=v \text { for all } t \geq 0
$$

In this case, we also say that the underlying probability measure on $\mathcal{N}$ is an equilibrium measure. Let $\alpha \in(\pi, 3 \pi / 2)$ and define the measure in $\mathcal{N}$

$$
v_{\alpha}((x, y])=B_{\alpha}((x, 0),(y, 0)) \text { for } x \leq y \in \mathbb{R} .
$$

Compare this to (3.4) $\left(v_{\alpha}=v_{0}^{\alpha}\right)$. It was this interplay between the longest path description and the equilibrium interacting particle system that proved very fruitful in the results for the classical Hammersley process in Cator and Groeneboom [6]. We will attempt the same in the interacting fluid system, but since the equilibrium solution is not explicitly known, we needed to develop new tools and ideas, which in fact also had interesting applications for the classical case. Of course, as an immediate consequence of Proposition 3.2, we do have the following

Corollary 4.4 The random measures $v_{\alpha}=v_{0}^{\alpha}$ (3.4) are all equilibrium measures for the Hammersley interacting fluid process. Furthermore, for all $(x, t) \in \mathbb{R} \times[0, \infty)$,

$$
L_{v_{\alpha}}(x, t)=B_{\alpha}((0,0),(x, t)) .
$$

\section{Ergodicity and uniqueness of the equilibrium measure}

To prove the next theorem about time invariance, we need to define the following exit points: for $t \geq 0$,

$$
\left(Z_{\alpha}(x, t), 0\right) \text { is the crossing point of } \varpi_{\alpha}(x, t) \text { and } \mathbb{R} \times\{0\} .
$$

This was already used in the proof of Proposition 3.2. Analogously, for $x \geq 0$,

$$
\left(0, Z_{\alpha}^{*}(x, t)\right) \text { is the crossing point of } \varpi_{\alpha}(x, t) \text { and }\{0\} \times \mathbb{R} \text {. }
$$

We have the following proposition.

Proposition 5.1 For all $x \in \mathbb{R}$ and $t \geq 0$, we have

$$
Z_{\alpha}(x, t)=Z_{v_{\alpha}}(x, t) .
$$

Proof From (3.3) and Corollary 4.4 we immediately get that $Z_{v_{\alpha}}(x, t) \geq Z_{\alpha}(x, t)$. Now suppose $Z_{v_{\alpha}}(x, t)>Z_{\alpha}(x, t)$. Since

$$
\begin{aligned}
B_{\alpha}((0,0),(x, t)) & =L_{v_{\alpha}}(x, t) \\
& =v_{\alpha}\left(Z_{v_{\alpha}}(x, t)\right)+L\left(\left(Z_{v_{\alpha}}(x, t), 0\right),(x, t)\right) \\
& =B_{\alpha}\left((0,0),\left(Z_{v_{\alpha}}(x, t), 0\right)\right)+L\left(\left(Z_{v_{\alpha}}(x, t), 0\right),(x, t)\right),
\end{aligned}
$$


we conclude that

$$
L\left(\left(Z_{v_{\alpha}}(x, t), 0\right),(x, t)\right)=B_{\alpha}\left(\left(Z_{v_{\alpha}}(x, t), 0\right),(x, t)\right) .
$$

Now define $\mathbf{c}$ as the coalescing point of the $\alpha$-ray starting at $\left(Z_{v_{\alpha}}(x, t), 0\right)$ and the $\alpha$-ray starting at $(x, t)$ (which goes through $\left.\left(Z_{\alpha}(x, t), 0\right)\right)$. Then

$$
\begin{aligned}
L(\mathbf{c},(x, t)) & =B_{\alpha}(\mathbf{c},(x, t)) \quad \text { (because the two points are on one } \alpha \text {-ray) } \\
& =B_{\alpha}\left(\mathbf{c},\left(Z_{v_{\alpha}}(x, t), 0\right)\right)+B_{\alpha}\left(\left(Z_{v_{\alpha}}(x, t), 0\right),(x, t)\right) \\
& =L\left(\mathbf{c},\left(Z_{v_{\alpha}}(x, t), 0\right)\right)+L\left(\left(Z_{v_{\alpha}}(x, t), 0\right),(x, t)\right),
\end{aligned}
$$

which would imply that there exists a longest path from $\mathbf{c}$ to $(x, t)$ which is strictly below the original $\alpha$-ray, contradicting the uniqueness of the lowest finite geodesic.

Since the $\alpha$-ray $\varpi_{\alpha}(\mathbf{0})$ has asymptotic direction $(\cos \alpha, \sin \alpha)$ with probability 1 , we can easily see that

$$
\lim _{t \rightarrow \infty} \mathbb{P}\left(\left|Z_{\alpha}(t,(\tan \alpha) t)\right| \geq \epsilon t\right)=\lim _{t \rightarrow \infty} \mathbb{P}\left(\left|Z_{\alpha}^{*}(t,(\tan \alpha) t)\right| \geq \epsilon t\right)=0
$$

This follows from the fact that $Z_{\alpha}(t,(\tan \alpha) t)$ has the same distribution as the crossing of $\varpi_{\alpha}(\mathbf{0})$ with the horizontal line $\mathbb{R} \times\{-(\tan \alpha) t\}$, and a similar relation holds for $Z_{\alpha}^{*}(t,(\tan \alpha) t)$.

However, we can also control $Z_{v}$ for more general $v$. Compare the following lemma to Lemma 3.3 in Ferrari, Martin and Pimentel [9].

Lemma 5.2 Suppose $v \in \mathcal{N}$. Assume that

$$
\liminf _{z \rightarrow-\infty} \frac{\nu((z, 0])}{-z} \geq \frac{\gamma}{2} \sqrt{\tan \alpha} \text { and that } \limsup _{z \rightarrow \infty} \frac{\nu((0, z])}{z} \leq \frac{\gamma}{2} \sqrt{\tan \alpha}
$$

Let $h \in \mathbb{R}$. Then, with probability one,

$$
\lim _{t \rightarrow \infty} \frac{Z_{v}(t+h,(\tan \alpha) t)}{t}=0 .
$$

Furthermore, define

$$
\tilde{Z}_{v, h}(t,(\tan \alpha) t)=\underset{z \leq t}{\operatorname{argmax}}\{v(z)+L((-t+z,-(\tan \alpha) t),(h, 0))\} .
$$

Here we take the right-most maximum. The idea is that we move the origin to $(-t,-(\tan \alpha) t)$ and look at the exit point for $(h, 0)$. Then, with probability one,

$$
\lim _{t \rightarrow \infty} \frac{\tilde{Z}_{v, h}(t,(\tan \alpha) t)}{t}=0
$$

The slightly technical proof of this lemma can be found in Appendix. 
Now we can prove the most important result of this section.

Theorem 5.3 The process $x \mapsto v_{\alpha}(x)$ is stationary and ergodic and its intensity is given by

$$
\mathbb{E} v_{\alpha}(1)=\frac{\gamma(F)}{2} \sqrt{\tan \alpha}
$$

Finally, consider a random $v \in \mathcal{N}$, which is time invariant, and which defines a stationary and ergodic process on $\mathbb{R}$ with $0<\mathbb{E}(\nu(1))<+\infty$. Define $\alpha \in(\pi, 3 \pi / 2)$ by

$$
\alpha=\arctan \left(\frac{2}{\gamma(F)} \mathbb{E} v(1)\right)^{2}
$$

Then $v \stackrel{\mathcal{D}}{=} v_{\alpha}$.

Proof The fact that $x \mapsto v_{\alpha}(x)$ is stationary and that $0<\mathbb{E}\left(v_{\alpha}(1)\right)<+\infty$ follows directly from Proposition 3.1. Ergodicity will follow from Proposition 5.6. Fix $\alpha \in(\pi, 3 \pi / 2)$ and set $\rho=\rho(\alpha):=\sqrt{\tan \alpha}$. Since the model is invariant under the $\operatorname{map}(x, t) \rightarrow(\rho x, t / \rho)$,

$$
\mathbb{E} v_{\alpha}(1)=\mathbb{E} \nu_{5 \pi / 4}(\rho)=\mathbb{E} \nu_{5 \pi / 4}(1) \sqrt{\tan \alpha}
$$

Now, for all $t \geq 0$,

$$
L_{v_{5 \pi / 4}}(t, t)=v_{5 \pi / 4}\left(Z_{v_{5 \pi / 4}}(t, t)\right)+L\left(\left(Z_{v_{5 \pi / 4}}(t, t), 0\right),(t, t)\right) .
$$

By Proposition 5.1, $Z_{v_{5 \pi / 4}}(t, t)=Z_{5 \pi / 4}(t, t)=: Z_{5 \pi / 4}(t)$. If $Z_{5 \pi / 4}(t) \geq 0$ then

$$
0 \leq L_{\nu_{5 \pi / 4}}(t, t)-L((0,0),(t, t)) \leq v_{5 \pi / 4}\left(Z_{5 \pi / 4}(t)\right)
$$

On the other hand, if $Z_{5 \pi / 4}(t)<0$ then $Z_{5 \pi / 4}^{*}(t):=Z_{v_{5 \pi / 4}}^{*}(t, t)=Z_{5 \pi / 4}^{*}(t, t) \geq 0$. It follows from (4.6) and Corollary 4.4 that

$$
v_{\alpha}^{*}(x)=B_{\alpha}(\mathbf{0},(0, x)) .
$$

From the additivity of the Busemann function, we know that

$$
L_{v_{5 \pi / 4}}(t, t)=v_{5 \pi / 4}^{*}\left(Z_{5 \pi / 4}^{*}(t, t)\right)+L\left(\left(0, Z_{5 \pi / 4}^{*}(t, t)\right),(t, t)\right) .
$$

Finally we obtain that,

$$
0 \leq L_{v_{5 \pi / 4}}(t, t)-L((0,0),(t, t)) \leq \max \left\{v_{5 \pi / 4}\left(Z_{5 \pi / 4}(t)\right), v_{5 \pi / 4}^{*}\left(Z_{5 \pi / 4}^{*}(t)\right)\right\} .
$$


Clearly, from Proposition 3.1(3) (symmetry) it follows that $v_{5 \pi / 4}^{*} \stackrel{\mathcal{D}}{=} v_{5 \pi / 4}$. Equation (5.3) implies that for any $\eta>0, \mathbb{P}\left(\max \left\{Z_{5 \pi / 4}(t), Z_{5 \pi / 4}^{*}(t)\right\} \geq \eta t\right) \rightarrow 0$ as $t \rightarrow \infty$. Let $\varepsilon>0$. The ergodicity of $v_{5 \pi / 4}$ (and of $v_{5 \pi / 4}^{*}$ ) and the fact that $\mathbb{E} v_{\alpha}(1)<+\infty$ imply that there exists $\eta>0$ such that for all $t$ big enough,

$$
\mathbb{P}\left(v_{5 \pi / 4}(\eta t) \geq \varepsilon t \quad \text { or } \quad v_{5 \pi / 4}^{*}(\eta t) \geq \varepsilon t\right) \leq \varepsilon / 2
$$

Again for $t$ big enough, we know that $\mathbb{P}\left(\max \left\{Z_{5 \pi / 4}(t), Z_{5 \pi / 4}^{*}(t)\right\} \geq \eta t\right) \leq \varepsilon / 2$. Combining all this gives, for $t$ big enough,

$$
\mathbb{P}\left(L_{v_{5 \pi / 4}}(t, t)-L((0,0),(t, t)) \geq \varepsilon t\right) \leq \varepsilon
$$

Therefore,

$$
\frac{L_{v_{5 \pi / 4}}(t, t)-L((0,0),(t, t))}{t} \stackrel{\mathcal{D}}{\longrightarrow} 0 .
$$

By Corollary 4.4 and using Proposition 3.1(3), we get

$$
\begin{aligned}
\mathbb{E} L_{v_{5 \pi / 4}}(t, t) & =\mathbb{E} B_{5 \pi / 4}(\mathbf{0},(t, t)) \\
& =\mathbb{E} B_{5 \pi / 4}(\mathbf{0},(t, 0))+\mathbb{E} B_{5 \pi / 4}((t, 0),(t, t)) \\
& =2 t \mathbb{E}\left(v_{5 \pi / 4}(1)\right) .
\end{aligned}
$$

The ergodic theorem applied to $B_{5 \pi / 4}(t, t)^{1}$ implies that, with probability one,

$$
\lim _{t \rightarrow \infty} \frac{B_{5 \pi / 4}(t, t)}{t}=2 \mathbb{E} \nu_{5 \pi / 4}(1) .
$$

Combining this with Theorem 2.1 and (5.8), one gets (5.6).

Now we need to address the uniqueness of $v_{\alpha}$. Suppose $v \in \mathcal{N}$ is ergodic and time invariant. Define $Z(t)=Z_{v}(t,(t \tan \alpha))$ and $Z_{h}(t)=Z_{v}(t+h,(\tan \alpha) t)$. Now define, as in Lemma 5.2,

$$
\tilde{Z}(t)=\underset{z \leq t}{\operatorname{argmax}}\{v(z)+L((-t+z,-(\tan \alpha) t), \mathbf{0})\}
$$

and

$$
\tilde{Z}_{h}(t)=\underset{z \leq t+h}{\operatorname{argmax}}\{v(z)+L((-t+z,-(\tan \alpha) t),(h, 0))\} .
$$

Here, we take the right-most location of the maximum. The intuition for $\tilde{Z}(t)$ and $\tilde{Z}_{h}(t)$ is that we place the origin at $(-t,-(\tan \alpha) t)$, and look at the exit-point for the

\footnotetext{
1 Ergodicity will follow from Proposition 5.6.
} 
path that starts at $(-t,-(\tan \alpha) t)$, picks up mass from $v$ and then goes to $\mathbf{0}$, resp. $(h, 0)$. Clearly, we have

$$
\left(Z(t), Z_{h}(t)\right) \stackrel{\mathcal{D}}{=}\left(\tilde{Z}(t), \tilde{Z}_{h}(t)\right)
$$

Since $v$ is ergodic, and by our choice of $\alpha$, v satisfies (5.4). Lemma 5.2 then tells us that

$$
\left(\tilde{Z}(t), \tilde{Z}_{h}(t)\right) / t \stackrel{\text { a.s. }}{\longrightarrow}(0,0) .
$$

This means that the two paths $\varpi((-t+\tilde{Z}(t),-t(\tan \alpha)), \mathbf{0})$ and $\varpi\left(\left(-t+\tilde{Z}_{h}(t)\right.\right.$, $-(\tan \alpha) t),(h, 0))$ will converge in any bounded box to the $\alpha$-rays $\varpi_{\alpha}(\mathbf{0})$ and $\varpi_{\alpha}((h, 0))$ respectively (this follows from Theorem 2.2(2)). However, these two $\alpha$ rays will coalesce, which means that with probability 1 , there exists $t_{0}>0$ such that for all $t \geq t_{0}$, the two converging paths coalesce, which in turn implies that $\tilde{Z}(t)=\tilde{Z}_{h}(t)$ (because they are both the right-most point where the maximum takes place and, as soon as they coalesce, they get the same exit point). Now define

$$
\tilde{L}(t)=\sup _{z \leq t}\{v(z)+L((-t+z,-(\tan \alpha) t), \mathbf{0})\}
$$

and

$$
\tilde{L}_{h}(t)=\sup _{z \leq t+h}\{v(z)+L((-t+z,-(\tan \alpha) t),(h, 0))\} .
$$

We also have that

$$
\left(\tilde{L}(t), \tilde{L}_{h}(t)\right) \stackrel{\mathcal{D}}{=}\left(L_{v}(t,(\tan \alpha) t), L_{v}(t+h,(\tan \alpha) t)\right) .
$$

Furthermore, if $t \geq t_{0}$, then

$$
\tilde{L}_{h}(t)-\tilde{L}(t)=B_{\alpha}(\mathbf{0},(h, 0))=v_{\alpha}((0, h]) .
$$

This proves that

$$
\begin{aligned}
M_{t(\tan \alpha)}^{v}((t, t+h]) & =L_{v}(t+h,(\tan \alpha) t)-L_{v}(t,(\tan \alpha) t) \\
& \stackrel{\mathcal{D}}{\longrightarrow} v_{\alpha}((0, h]) .
\end{aligned}
$$

Since $v$ is time invariant and ergodic, we see that

$$
v((0, h]) \stackrel{\mathcal{D}}{=} M_{(\tan \alpha) t}^{v}((t, t+h]) \stackrel{\mathcal{D}}{=} v_{\alpha}((0, h]) .
$$

In principle, we need to show convergence for a finite number of $h$ 's simultaneously, but it is not hard to see that the ideas we used can be extended to that case, at the 
cost of some notational burden. Note that we have proved that for any deterministic $v$ satisfying (5.4), $M_{(\tan \alpha) t}^{v}([t, t+h])$ converges in distribution to $v_{\alpha}(h)$, as a process in $h$. This shows that in a rarefaction fan, the fluid process converges locally to the correct equilibrium process (local equilibrium).

\section{Corollary 5.4}

$$
\mathbb{E}\left(v_{\alpha}^{*}(1)\right)=\frac{\gamma(F)}{2 \sqrt{\tan \alpha}} .
$$

In particular, for all $\alpha \in(\pi, 3 \pi / 2)$, we have

$$
\mathbb{E}\left(v_{\alpha}(1)\right) \cdot \mathbb{E}\left(v_{\alpha}^{*}(1)\right)=\frac{\gamma(F)^{2}}{4} .
$$

Proof Remember that $\nu_{\alpha}^{*}(x)=B_{\alpha}(\mathbf{0},(0, x))$. For $\alpha=5 \pi / 4$, the result follows Proposition 3.1(3) (symmetry) and Theorem 5.3. Now use the map $(x, t) \mapsto(\rho x, t / \rho)$ to see that

$$
v_{\alpha}^{*}(x) \stackrel{\mathcal{D}}{=} v_{5 \pi / 4}^{*}(x / \sqrt{\tan \alpha})
$$

For the classical Hammersley model, we know that if $\bar{\nu}_{\lambda}$ is a Poisson counting process of intensity $\lambda$, then $\bar{\nu}_{\lambda}$ is time invariant and ergodic. Therefore it must be equal in distribution to $v_{\alpha}$, for some $\alpha \in(\pi, 3 \pi / 2)$. Choose $\lambda=1$ and define $\alpha_{1} \in(\pi, 3 \pi / 2)$ such that $\bar{\nu}_{1} \stackrel{\mathcal{D}}{=} v_{\alpha_{1}}$. We know that in the classical Hammersley process,

$$
L_{\bar{v}_{1}}(0, t) \stackrel{\mathcal{D}}{=} \bar{v}_{1}((0, t]) .
$$

This means that $v_{\alpha_{1}}(t) \stackrel{\mathcal{D}}{=} v_{\alpha_{1}}^{*}(t)$. We can now use Theorem 5.3 and Corollary 5.4 to conclude that $\alpha_{1}=5 \pi / 4$ (since we must have that $\tan \alpha_{1}=1$ ). Consequently,

$$
1=\mathbb{E} \bar{\nu}_{1}(1)=\mathbb{E} \nu_{5 \pi / 4}(1)=\frac{\gamma\left(\delta_{1}\right)}{2} \sqrt{\tan (5 \pi / 4)}=\frac{\gamma\left(\delta_{1}\right)}{2},
$$

which proves that $\gamma\left(\delta_{1}\right)=2$. We remark that the proof that the Poisson process is time invariant does not depend on the value of $\gamma\left(\delta_{1}\right)$. It only relies on an explicit calculation of the generator associated to $M_{t}$. (See Lemma 8 of [1], or Theorem 3.1 of [6]).

Corollary 5.5 In the classical Hammersley model, we have that $\gamma(1)=2$ and that $\nu_{\alpha} \stackrel{\mathcal{D}}{=} \bar{v}_{\lambda(\alpha)}$ where $\lambda(\alpha)=\sqrt{\tan \alpha}$.

For general weight distributions $F$, we were not able to get more information on $v_{\alpha}$ (not even a guess for a good candidate). In particular, we do not know how to calculate $\gamma(F)$. This does seem to be the most important contribution of the interacting fluid representation: once we have a good candidate for $v_{\alpha}$, we can check it by showing that it is invariant under the evolution of the interacting fluid. In fact, even in the results 
for the classical Hammersley case found in Aldous and Diaconis [1] and Cator and Groeneboom $[5,6]$, this is where the interacting particle process proves its worth.

\subsection{Mixing property of $v_{\alpha}$}

We will show that the measure $v_{\alpha}$ has the following mixing property, usually called strong mixing in dynamical systems. We consider the $\sigma$-algebra $\mathcal{F}=\sigma\left\{v_{\alpha}((a, b])\right.$ : $a \leq b \in \mathbb{R}\}$ on the sample space $\Omega$, defined by the compound Poisson process. We can define the translation $\tau_{t}$ as an $\mathcal{F}$-measurable map from $\Omega$ to $\Omega$, simply by translating all Poisson points by the vector $(t, 0)$.

Proposition 5.6 For each $\alpha \in(\pi, 3 \pi / 2), v_{\alpha}$ satisfies

$$
\forall A, B \in \mathcal{F}: \lim _{t \rightarrow \infty} \mathbb{P}\left(A \cap \tau_{t}^{-1}(B)\right)=\mathbb{P}(A) \mathbb{P}(B) .
$$

In particular, this implies that $v_{\alpha}$ is ergodic.

Proof From translation invariance and a standard approximation of sets in $\mathcal{F}$, it is enough to prove (5.9) for all $A, B \in \mathcal{F}_{h}:=\sigma\left\{v_{\alpha}((a, b]): a \leq b \in[0, h]\right\}$. Consider the paths $\varpi((-t,-t(\tan \alpha)), \mathbf{0})$ and $\varpi((-t,-t(\tan \alpha)),(h, 0))$. Almost surely, these paths will converge to $\varpi_{\alpha}(\mathbf{0})$ and $\varpi_{\alpha}((h, 0))$, respectively, on any finite box. This means, that if we define for $a, b \in[0, h]$

$$
v_{\alpha}^{(t)}((a, b])=L((b, 0),(-t,-t(\tan \alpha)))-L((a, 0),(-t,-t(\tan \alpha))),
$$

then for $t$ big enough, we have $v_{\alpha}^{(t)}=\left.v_{\alpha}\right|_{[0, h]}$. Clearly, $\tau_{t+h}^{-1}(B)$ is independent of $v_{\alpha}^{(t)}$, since they depend on the Poisson process to the left respectively to the right of the line $\{-t\} \times \mathbb{R}$. Define the event

$$
C_{t}=\left\{\forall s \geq t: v_{\alpha}^{(s)}=\left.v_{\alpha}\right|_{[0, h]}\right\}
$$

and denote $A^{(t)}$ the counterpart of the event $A$ in $\mathcal{F}_{h}^{(t)}:=\sigma\left\{v_{\alpha}^{(t)}((a, b]): a \leq b \in\right.$ $[0, h]\}$; although it is intuitively clear what is meant, we will make this more precise at the end of the proof. Then

$$
\begin{aligned}
\left|\mathbb{P}\left(A \cap \tau_{t+h}^{-1}(B)\right)-\mathbb{P}(A) \mathbb{P}(B)\right| & \leq\left|\mathbb{P}\left(A \cap \tau_{t+h}^{-1}(B) \cap C_{t}\right)-\mathbb{P}(A) \mathbb{P}(B)\right|+\mathbb{P}\left(C_{t}^{c}\right) \\
& =\left|\mathbb{P}\left(A^{(t)} \cap \tau_{t+h}^{-1}(B) \cap C_{t}\right)-\mathbb{P}(A) \mathbb{P}(B)\right|+\mathbb{P}\left(C_{t}^{c}\right) \\
& \leq\left|\mathbb{P}\left(A^{(t)} \cap \tau_{t+h}^{-1}(B)\right)-\mathbb{P}(A) \mathbb{P}(B)\right|+2 \mathbb{P}\left(C_{t}^{c}\right) \\
& =\mathbb{P}(B)\left|\mathbb{P}\left(A^{(t)}\right)-\mathbb{P}(A)\right|+2 \mathbb{P}\left(C_{t}^{c}\right) \\
& \leq 4 \mathbb{P}\left(C_{t}^{c}\right) .
\end{aligned}
$$

The proposition now follows from the fact that ${ }^{2} \mathbb{P}\left(C_{t}^{c}\right) \rightarrow 0$.

\footnotetext{
2 We note that, in the classical model, we have independent increments even if the probability of the event $C_{t}^{c}$ does not decay to 0 very fast. This indicates that, to show mixing by using these events may not be the best strategy.
} 
To see what is meant by $A^{(t)}$, we define the index-set $I=\left\{v_{\alpha}((a, b]): a \leq b \in\right.$ $[0, h]\}$ and $I^{(t)}=\left\{v_{\alpha}^{(t)}((a, b]): a \leq b \in[0, h]\right\}$. There is a canonical bijection $i: I \rightarrow I^{(t)}$. Define $\mathcal{B}$ as the product $\sigma$-algebra on $\mathbb{R}^{I}$, and likewise $\mathcal{B}^{(t)}$. Extend the canonical map $i$ such that $i: \mathbb{R}^{I} \rightarrow \mathbb{R}^{I^{(t)}}$. Define the map

$$
\phi: \Omega \rightarrow \mathbb{R}^{I}: \omega \mapsto\left\{v_{\alpha}((a, b])(\omega): a \leq b \in[0, h]\right\}
$$

and likewise $\phi_{t}: \Omega \rightarrow \mathbb{R}^{I^{(t)}}$. We know that $\mathcal{F}_{h}=\phi^{-1}(\mathcal{B})$ and $\mathcal{F}_{h}^{(t)}=\phi_{t}^{-1}\left(\mathcal{B}^{(t)}\right)$. This means that there exists $U \in \mathcal{B}$, such that $A=\phi^{-1}(U)$. We define $A^{(t)}=\phi_{t}^{-1}(i(U))$.

\section{Central limit theorems for the Busemann function in the classical model}

Our geometrical approach yields a very explicit description of the fluctuations of the Busemann function in the classical Hammersley model. We first notice the following relations that will be derived from Proposition 3.1 and Proposition 3.2:

Proposition 6.1 Consider the classical Hammersley last-passage model with weights equal to 1 and recall $\vec{\beta}:=(\cos \beta, \sin \beta)$.

- If $\beta \in[0, \pi]$ then, as processes,

$$
B_{\alpha}(\mathbf{0}, \cdot \vec{\beta})=L_{v_{\alpha}}(\cdot \vec{\beta})
$$

- Consider two independent one-dimensional Poisson processes $X_{\alpha}$ and $Y_{\alpha}$ with intensity $\lambda(\alpha)$ and $1 / \lambda(\alpha)$, respectively. Here, $\lambda(\alpha)=\sqrt{\tan \alpha}$. If $\beta \in[\pi / 2, \pi]$ then, as processes for $t \geq 0$,

$$
B_{\alpha}(\mathbf{0}, t \vec{\beta}) \stackrel{\mathcal{D}}{=} Y_{\alpha}(t \sin \beta)-X_{\alpha}(-t \cos \beta) .
$$

Proof The first statement follows directly from Proposition 3.2. To obtain the second relation, note that, by additivity and anti-symmetry (Proposition 3.1),

$$
\begin{aligned}
B_{\alpha}(\mathbf{0}, t \vec{\beta}) & =B_{\alpha}(\mathbf{0},(t \cos \beta, 0))+B_{\alpha}((t \cos \beta, 0), t \vec{\beta}) \\
& =B_{\alpha}((t \cos \beta, 0), t \vec{\beta})-B_{\alpha}((t \cos \beta, 0), \mathbf{0}) \\
& =L_{v_{\alpha}}((t \cos \beta, 0),(t \cos \beta, t \sin \beta))-L_{v_{\alpha}}((t \cos \beta, 0), \mathbf{0}) .
\end{aligned}
$$

In the classical Hammersley process we have a version of Burke's Theorem (see Theorem 3.1 in [5]), which implies that both $t \mapsto L_{v_{\alpha}}(\mathbf{0},(t, 0))$ and $t \mapsto L_{v_{\alpha}}(\mathbf{0},(0, t))$ are Poisson processes with intensity $\lambda(\alpha)$ and $1 / \lambda(\alpha)$ respectively, and that $t \mapsto$ $L_{v_{\alpha}}(\mathbf{0}, t \vec{\beta})$ has independent increments. This last statement can be seen in the following way: consider the increments $L_{v_{\alpha}}(\mathbf{0}, s \vec{\beta})$ and $L_{v_{\alpha}}(s \vec{\beta}, t \vec{\beta})$, for $0 \leq s \leq t$; see Fig. 2.

Then both increments depend on the independent Poisson processes $L_{v_{\alpha}}$ restricted to (the line segments) $\mathbf{0} A$ and $A B$, and on the Poisson process in the triangle $\mathbf{0} A B$. 
Fig. 2 Independent increments for $L_{v_{\alpha}}$

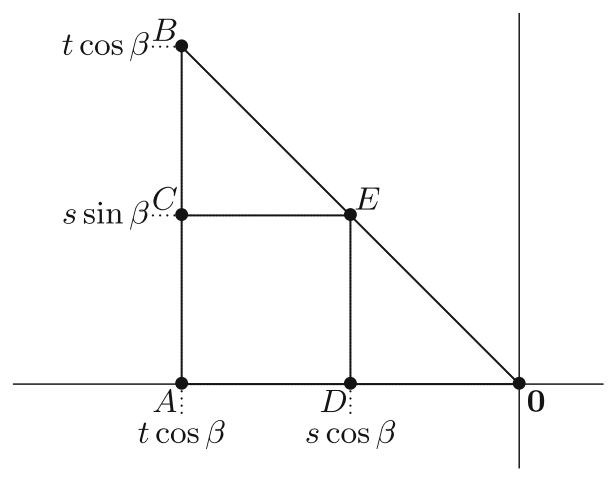

Burke's Theorem for $L_{v_{\alpha}}$ now implies that $L_{v_{\alpha}}$ restricted to $C E$ is independent of $L_{v_{\alpha}}$ restricted to $E D$. Since $L_{v_{\alpha}}$ restricted to $B E$ depends on $L_{v_{\alpha}}$ on $C E, C B$ and the Poisson process in $E C B$, whereas $L_{v_{\alpha}}$ restricted to $\mathbf{0} E$ depends on $L_{v_{\alpha}}$ on $\mathbf{0} D, D E$ and the Poisson process in $\mathbf{0} D E$, and since these six processes are all independent, we proved that the increments are independent. This proves the Proposition.

In the classical set-up, Corollary 5.5, together with Proposition 6.1, implies that:

(1) if $\beta=0$ then

$$
B_{\alpha}(\mathbf{0}, \cdot \vec{\beta}) \stackrel{\mathcal{D}}{=} X_{\alpha}(\cdot)
$$

where $X_{\alpha}$ is a Poisson process of intensity $\lambda(\alpha)$.

(2) if $\beta \in(0, \pi / 2)$ then

$$
B_{\alpha}(\mathbf{0}, \cdot \vec{\beta}) \stackrel{\mathcal{D}}{=} L_{\bar{v}_{\lambda(\alpha)}}(\cdot \vec{\beta})
$$

where $\lambda=\lambda(\alpha)=\sqrt{\tan \alpha}$

(3) if $\beta \in[\pi / 2, \pi]$ then,

$$
B_{\alpha}(\mathbf{0}, \cdot \vec{\beta}) \stackrel{\mathcal{D}}{=} Y_{\alpha}(\cdot \sin \beta)-X_{\alpha}(-\cdot \cos \beta)
$$

where $Y_{\alpha}$ and $X_{\alpha}$ are two independent one-dimensional Poisson processes variables of intensity $1 / \lambda(\alpha)$ and $\lambda(\alpha)$, respectively.

Baik and Rains [2] proved the following central limit theorem for the Hammersley classical model with external "sources". Let $\Phi(x)$ be the standard normal distribution function, and let $F_{0}(x)$ be the zero mean Tracy-Widom type distribution function introduced in Definition 2 of [2].

(4) If $\lambda \in(0,1)$ then

$$
\lim _{s \rightarrow \infty} \mathbb{P}\left(\frac{L_{\bar{v}_{\lambda}}(s, s)-(1 / \lambda+\lambda) s}{(\sqrt{1 / \lambda-\lambda}) s^{1 / 2}} \leq x\right)=\Phi(x) ;
$$


(5) If $\lambda=1$ then

$$
\lim _{s \rightarrow \infty} \mathbb{P}\left(\frac{L_{\bar{v}_{\lambda}}(s, s)-2 s}{s^{1 / 3}} \leq x\right)=F_{0}(x)
$$

(6) If $\lambda>1$ then

$$
\lim _{s \rightarrow \infty} \mathbb{P}\left(\frac{L_{\bar{v}_{\lambda}}(s, s)-(\lambda+1 / \lambda) s}{(\sqrt{\lambda-1 / \lambda}) s^{1 / 2}} \leq x\right)=\Phi(x) .
$$

These results naturally lead us to a central limit theorem for the Busemann function

$$
B(\beta, \cdot):=B_{5 \pi / 4}(\mathbf{0},(\cdot \cos \beta, \cdot \sin \beta)) .
$$

By (2.4), w.l.o.g. we can restrict ourselves to $\alpha=5 \pi / 4$. In this case we have

$$
\mathbb{E} B(\beta, t)=(\cos \beta+\sin \beta) t .
$$

Corollary 6.2 Consider the Hammersley classical last-passage model. Then

$$
\lim _{t \rightarrow \infty} \mathbb{P}\left(\frac{B(\pi / 4, t)-\sqrt{2} t}{2^{-1 / 6} t^{1 / 3}} \leq x\right)=F_{0}(x),
$$

while for $\beta \in[0, \pi / 4)$

$$
\lim _{t \rightarrow \infty} \mathbb{P}\left(\frac{B(\beta, t)-(\cos \beta+\sin \beta) t}{(\sqrt{\cos \beta-\sin \beta}) t^{1 / 2}} \leq x\right)=\Phi(x),
$$

and for $\beta \in(\pi / 4, \pi]$

$$
\lim _{t \rightarrow \infty} \mathbb{P}\left(\frac{B(\beta, t)-(\cos \beta+\sin \beta) t}{(\sqrt{\sin \beta-\cos \beta}) t^{1 / 2}} \leq x\right)=\Phi(x) .
$$

Proof The statement for $\beta=0$ and $\beta \in[\pi / 2, \pi]$ follows (1) and (3), together with the central limit theorem. Now, let us take $\beta \in(0, \pi / 2)$. Consider the map (2.4) with $\rho(\beta)=\sqrt{\tan \beta}$. Then $B(\beta, t) \stackrel{\mathcal{D}}{=} L_{\bar{v}_{\rho}}(s, s)$, where $s=(\sqrt{\sin \beta \cos \beta}) t$, and for $\beta \in(0, \pi / 4]$ we have $\rho \in(0,1]$ while for $\beta \in(\pi / 4, \pi]$ we have $\rho \in(1, \infty)$.

\subsection{The crossing formula}

In the classical model, the exit point formula for the equilibrium regime, proved by Cator and Groeneboom [6], is

$$
\operatorname{Var} L_{\bar{v}_{\lambda}}(x, t)=-\lambda x+\frac{t}{\lambda}+2 \lambda \mathbb{E} Z_{\bar{v}_{\lambda}}(x, t)_{+},
$$


where $\operatorname{Var} X$ is the variance of $X$, and $X_{+}:=\max \{X, 0\}$. Together with Propositions 5.1 and 6.1, this relates the variance of the Busemann function at $(x, t)$ with the position of the crossing point of the $\alpha$-ray starting at $(x, t)$ :

$$
\operatorname{Var} B_{\alpha}(\mathbf{0},(x, t))=-(\sqrt{\tan \alpha}) x+\frac{t}{\sqrt{\tan \alpha}}+2(\sqrt{\tan \alpha}) \mathbb{E} Z_{\alpha}(x, t)_{+} .
$$

In particular,

$$
\operatorname{Var} B_{5 \pi / 4}(\mathbf{0},(t, t))=2 \mathbb{E} Z_{5 \pi / 4}(t, t)_{+}
$$

We note that this crossing point formula can be seen as a version of the scaling identity $\xi=2 \chi$, where $\chi$ and $\xi$ are the critical exponents that measure the order of magnitude of the fluctuations of Busemann functions and crossing points, respectively.

\section{The multi-class process and second class particles}

For two positive measures $v$ and $\bar{v}$ on $\mathbb{R}$, we say that $\bar{v}$ dominates $v$, notation $\bar{v} \geq v$, whenever $\bar{v}(I) \geq v(I)$ for all measurable $I \subseteq \mathbb{R}$.

Proposition 7.1 Suppose we have two measures $v, \bar{v} \in \mathcal{N}$ such that $\bar{v} \geq v$. Define the corresponding interacting fluid system as $M_{t}^{v}$ and $M_{t}^{\bar{v}}$, using the same weighted Poisson process (basic coupling). Then $M_{t}^{\bar{v}} \geq M_{t}^{\nu}$ (as measures). If $\left.\bar{v}\right|_{(-\infty, 0)}=\left.v\right|_{(-\infty, 0)}$, then $M_{t}^{\bar{v}}([0, x])-M_{t}^{v}([0, x])$ is non-increasing in $t$ for all $x \geq 0$.

Proof Fix an interval $[-K, K]$ and a time $t$. There exists (a random) $M>0$ such that $M_{t}^{v}$ and $M_{t}^{\bar{v}}$ restricted to $[-K, K]$ only depend on Poisson points in $[-M, K] \times[0, t]$ and on $v$ and $\bar{v}$ restricted to $[-M, K]$ (it is not hard to see that we can take $M=$ $Z_{\nu}(-K, t)$ (see (4.9)). This means that we are only dealing with a finite number of Poisson points, so if we can prove that the premise " $M_{s}^{\bar{\nu}} \geq M_{s}^{v}$ for all $s<t$ " implies that $M_{t}^{\bar{\nu}} \geq M_{t}^{\nu}$, we will have proved the first statement, since it is obviously true for $t=0$. Suppose there exists a Poisson point at $\left(x_{0}, t\right)$ with weight $\omega$ for some $x_{0} \in[-M, K]$, since otherwise the implication is immediate. We then know, using Proposition 4.1 and (4.2), that if $x_{0}<x \leq y$,

$$
\begin{aligned}
M_{t}^{\nu}((x, y]) & =\left(M_{t-}^{\nu}\left(\left(x_{0}, y\right]\right)-\omega\right)_{+}-\left(M_{t-}^{\nu}\left(\left(x_{0}, x\right]\right)-\omega\right)_{+} \\
& \leq\left(M_{t-}^{\bar{v}}\left(\left(x_{0}, y\right]\right)-\omega\right)_{+}-\left(M_{t-}^{\bar{\nu}}\left(\left(x_{0}, x\right]\right)-\omega\right)_{+} \\
& =M_{t}^{\bar{v}}((x, y]) .
\end{aligned}
$$

The inequality follows from the fact that if $A \geq B$ and $\tilde{A} \geq \tilde{B} \geq 0$, then $(A-\omega)_{+}-$ $(B-\omega)_{+} \leq(A+\tilde{A}-\omega)_{+}-(B+\tilde{B}-\omega)_{+}$. If $x \leq x_{0}<\bar{y}$ or $x \leq y \leq x_{0}$, the implication is straightforward, following a similar split up. 
The second statement follows from a similar reasoning: suppose there is a Poisson point at $\left(x_{0}, t\right)$ with weight $\omega$. If $x>x_{0} \geq 0$,

$$
\begin{aligned}
M_{t}^{\bar{\nu}}\left(\left(x_{0}, x\right]\right)-M_{t}^{\nu}\left(\left(x_{0}, x\right]\right) & =\left(M_{t-}^{\bar{\nu}}\left(\left(x_{0}, x\right]\right)-\omega\right)_{+}-\left(M_{t-}^{\nu}\left(\left(x_{0}, x\right]\right)-\omega\right)_{+} \\
& \leq M_{t-}^{\bar{\nu}}\left(\left(x_{0}, x\right]\right)-M_{t-}^{\nu}\left(\left(x_{0}, x\right]\right)
\end{aligned}
$$

The inequality follows from the fact that $(A-c)_{+}-(B-c)_{+} \leq A_{+}-B_{+}$whenever $c \geq 0$ and $A \geq B$. Since $M_{t}^{\bar{v}}\left(\left[0, x_{0}\right]\right)-M_{t}^{v}\left(\left[0, x_{0}\right]\right)=M_{t-}^{\bar{\nu}}\left(\left[0, x_{0}\right]\right)-M_{t-}^{v}\left(\left[0, x_{0}\right]\right)$, this shows that

$$
M_{t}^{\bar{\nu}}([0, x])-M_{t}^{\nu}([0, x]) \leq M_{t-}^{\bar{v}}([0, x])-M_{t-}^{\nu}([0, x]) .
$$

Now suppose $x_{0}<0$ and $x \geq 0$. Note that under the condition on $\bar{v}$, we have that for all $s \geq 0$ and all $\varepsilon>0, L_{\bar{v}}(-\varepsilon, s)=L_{\nu}(-\varepsilon, s)$, so

$$
M_{s}^{\bar{\nu}}([0, x])-M_{s}^{\nu}([0, x])=L_{\bar{\nu}}(x, s)-L_{v}(x, s) .
$$

When $L_{\bar{v}}(x, t)$ does not use the weight at $\left(x_{0}, t\right)$, we know that $L_{\bar{v}}(x, t)=L_{\bar{v}}(x, t-)$ and that $L_{v}(x, t) \geq L_{v}(x, t-)$, which implies the desired result. If $L_{\bar{v}}(x, t)$ does use the weight at $\left(x_{0}, t\right)$, then it is not hard to see that $L_{v}(x, t)$ will also use the weight at $\left(x_{0}, t\right)$ (the longest path corresponding to $\bar{v}$ is always to the right of the path corresponding to $v$ ), which means that only the mass on the $x$-axis strictly to the left of 0 is used, and therefore $M_{t}^{\bar{v}}([0, x])=M_{t}^{\nu}([0, x])$. Finally, when $x_{0}=x$, we get that $M_{t}^{\bar{\nu}}([0, x])=M_{t-}^{\bar{\nu}}([0, x])+\omega$ and $M_{t}^{v}([0, x])=M_{t-}^{v}([0, x])+\omega$, and when $x_{0}>x$, we have $M_{t}^{\bar{v}}([0, x])=M_{t-}^{\bar{v}}([0, x])$ and $M_{t}^{v}([0, x])=M_{t-}^{v}([0, x])$.

In other words, Proposition 7.1 tells us that the interacting fluid system is monotone: if one starts the fluid process with the same Poisson weights (basic coupling) and with ordered initial configurations, then the order is preserved for all $t \geq 0$. This coupled process is called the multi-class fluid system. The multi-class system is just a convention to describe a coupled process with ordered initial configurations (Ferrari and Martin [8]).

\subsection{The multi-class invariant process}

With Theorem 2.2 in hands, for any countable $D \subseteq(\pi, 3 \pi / 2)$, one can construct simultaneously a collection of equilibrium processes $\left\{v_{\alpha}: \alpha \in D\right\}$ by using the same Poisson weights on $\mathbb{R} \times \mathbb{R}_{-}$and the Busemann functions $B_{\alpha}$. It turns out that this collection respects the order induced by the angles $\alpha \in D$. More precisely:

Theorem 7.2 If $\bar{\alpha}>\alpha$ then $v_{\bar{\alpha}} \geq v_{\alpha}$. In particular, for any countable subset $\left\{\alpha_{i}: i \in\right.$ $\mathbb{Z}\} \subseteq D$, if one runs simultaneously (basic coupling) the interacting fluid processes on $\mathbb{R} \times \mathbb{R}_{+}$with initial measures $\left(v_{\alpha_{i}}: i \in \mathbb{Z}\right)$ then, whenever $\alpha_{i}>\alpha_{j}, M_{t}^{v_{\alpha_{i}}} \geq M_{t}^{v_{\alpha_{j}}}$ for all $t \geq 0$, and

$$
\left(M_{t}^{v_{\alpha_{i}}}: i \in \mathbb{Z}\right) \stackrel{\mathcal{D}}{=}\left(v_{\alpha_{i}}: i \in \mathbb{Z}\right)
$$


Proof Let $z^{\prime} \geq z \in \mathbb{R}$. Let $\mathbf{m}$ be the crossing point between $\varpi_{\bar{\alpha}}((z, 0))$ and $\varpi_{\alpha}\left(\left(z^{\prime}, 0\right)\right)$. Furthermore, denote $\mathbf{c}$ as the coalescence point of the two $\alpha$-rays $\varpi_{\alpha}((z, 0))$ and $\varpi_{\alpha}\left(\left(z^{\prime}, 0\right)\right)$, and denote $\overline{\mathbf{c}}$ as the coalescence point of the two $\bar{\alpha}$-rays $\varpi_{\bar{\alpha}}((z, 0))$ and $\varpi_{\bar{\alpha}}\left(\left(z^{\prime}, 0\right)\right)$. Then

$$
\begin{aligned}
v_{\bar{\alpha}}\left(\left[z, z^{\prime}\right]\right)-v_{\alpha}\left(\left[z, z^{\prime}\right]\right)= & \left\{L\left(\overline{\mathbf{c}},\left(z^{\prime}, 0\right)\right)-L(\overline{\mathbf{c}},(z, 0))\right\} \\
& -\left\{L\left(\mathbf{c},\left(z^{\prime}, 0\right)\right)-L(\mathbf{c},(z, 0))\right\} \\
= & L\left(\overline{\mathbf{c}},\left(z^{\prime}, 0\right)\right)-\left\{L(\overline{\mathbf{c}}, \mathbf{m})+L\left(\mathbf{m},\left(z^{\prime}, 0\right)\right)\right\} \\
& +L(\mathbf{c},(z, 0))-\{L(\mathbf{c}, \mathbf{m})+L(\mathbf{m},(z, 0))\} \geq 0 .
\end{aligned}
$$

Notice that the Busemann functions $B_{\alpha}$ are a function of the compound Poisson process $(\mathbf{P}, \mathbf{w}): B_{\alpha}(\cdot, \cdot)=B_{\alpha}(\cdot, \cdot)(\mathbf{P}, \mathbf{w})$ (here $\mathbf{w}$ denotes the weights). Since $\mathbf{p}+\mathbf{P}$, the translated version of $\mathbf{P}$, has the same distribution as $\mathbf{P}$, we get that

$$
\left\{B_{\alpha_{i}}(\cdot, \cdot)(\mathbf{P}, \mathbf{w}): i \in \mathbb{Z}\right\} \stackrel{\mathcal{D}}{=}\left\{B_{\alpha_{i}}(\cdot, \cdot)(\mathbf{p}+\mathbf{P}, \mathbf{w}): i \in \mathbb{Z}\right\} .
$$

This shows time invariance for the Busemann multi-class process.

This result is also new in the classical Hammersley interacting system, where a different and explicit description of the invariant process with a finite number of classes is given in Ferrari and Martin [8].

\subsection{Law of large numbers for second-class particles}

Proposition 7.1 can be used to define the notion of second-class particles. In the interacting fluid system we can define it analogously to the interacting particle case, with a slight adaptation due to the continuous weights. We start by changing $v$ into $\bar{v}$, by putting an extra weight $\varepsilon>0$ in 0 , so

$$
\bar{v}([0, x])=v([0, x])+\varepsilon \text { for } x \geq 0 .
$$

With this new process, and using the same Poisson weights, we define $M_{t}^{\bar{\nu}}$. Clearly,

$$
M_{t}^{\bar{v}}([0, x]) \leq M_{t}^{\nu}([0, x])+\varepsilon .
$$

Now define the location of the second class particle $X_{v}(t)$ as

$$
X_{v}(t)=\inf \left\{x \geq 0: M_{t}^{\bar{v}}([0, x])=M_{t}^{v}([0, x])+\varepsilon\right\}
$$

By Proposition 7.1, $X_{v}(t)$ is a non-decreasing function of $t$, meaning that the second class particle moves to the right. In fact, the extra mass $\varepsilon$ will spread out, and our definition coincides with the rightmost point of this spread-out mass. This is a natural choice, since we will show that it does not depend on the total mass $\varepsilon$, while for example the leftmost point does depend on $\varepsilon$. 
There is the following important connection between the longest path description and the second class particle. Let $v^{+}$be the process defined by $v^{+}(x)=v(x)$ for $x \geq 0$, and by $v^{+}(x)=-\infty$ for $x<0$. We also define the process $v^{-}$by setting $v^{-}(x)=0$ for $x \geq 0$, and $v^{-}(x)=v(x)$ for $x<0$. Then

$$
L_{v^{+}}(x, t)= \begin{cases}\sup \{L((z, 0),(x, t))+v(z): 0 \leq z \leq x\} & \text { if } x \geq 0 \\ -\infty & \text { if } x<0\end{cases}
$$

and

$$
L_{v^{-}}(x, t)=\sup \{L((z, 0),(x, t))+v(z): z<0 \text { and } z \leq x\} .
$$

Clearly,

$$
L_{v}(x, t)=\max \left\{L_{v^{+}}(x, t), L_{v^{-}}(x, t)\right\} .
$$

Now suppose $x \geq 0$. If $L_{v^{+}}(x, t) \geq L_{v^{-}}(x, t)$, there exists a longest path that does not use any weight of $v$ on $(-\infty, 0)$. This means that if we add a weight $\varepsilon>0$ in the origin, $L_{\bar{v}}(x, t)=L_{\bar{v}^{+}}(x, t)=L_{v}(x, t)+\varepsilon$. Using Proposition 4.1, we see that this means that $M_{t}^{\bar{\nu}}(x)=M_{t}^{\nu}(x)+\varepsilon$, so $X_{v}(t) \leq x$. If on the other hand we start with $X_{v}(t) \leq x$, we conclude that $M_{t}^{\bar{\nu}}(x)=M_{t}^{\nu}(x)+\varepsilon$, using Proposition 7.1 and the fact that $M_{t}^{v}$ and $M_{t}^{\bar{v}}$ are right-continuous. This in turn means that $L_{\bar{v}}(x, t)=L_{v}(x, t)+\varepsilon$, which is only possible if $L_{v^{+}}(x, t) \geq L_{v^{-}}(x, t)$. We have shown that

$$
\left\{X_{v}(t) \leq x\right\}=\left\{L_{v^{+}}(x, t) \geq L_{v^{-}}(x, t)\right\} .
$$

Note that this can be rewritten as

$$
\left\{X_{v}(t) \leq x\right\}=\left\{Z_{v}(x, t) \geq 0\right\}
$$

This means that the path of the second class particle corresponds to a competition interface, a fact well known for the totally asymmetric exclusion process (Ferrari and Pimentel [10]). This allows us to show that the second class particle satisfies a strong law whenever $v^{+}$and $v^{-}$have asymptotic intensities. The proof of this does not use a coupling of two invariant versions of the fluid process, as is usual in the interacting particle case, but it uses the longest path description in a direct way. We would like to point out that in our general set-up, with random weights on the Poisson points, we do not have an equivalent of Burke's Theorem. This means that the time-reversed process is not a Hammersley interacting fluid system. Therefore, the path of a second class particle in general does not coincide in law with a longest path in the interacting fluid system, in contrast to the classical case, where the statement is true. However, we do have the following connection.

Proposition 7.3 Assume that the distribution of $v$ is translation invariant. Then, for any $t \geq 0$, we have that

$$
X_{v}(t)-x \stackrel{\mathcal{D}}{=}-Z_{v}(x, t)
$$


Proof This follows almost immediately from (7.2), since that equality can be rewritten as

$$
\left\{X_{v}(t)-x \leq h\right\}=\left\{Z_{v}(x+h, t) \geq 0\right\}
$$

Now use translation invariance to see that

$$
Z_{v}(x+h, t) \stackrel{\mathcal{D}}{=} Z_{v}(x, t)+h .
$$

Combining these two equations proves the proposition.

When we consider all $\alpha$-rays starting at the line $\mathbb{R} \times\{t\}$ and we move from left to right, $\left(X_{v_{\alpha}}(t), t\right)$ is the first point where the $\alpha$-ray passes the origin. It is tempting to think that the $\alpha$-ray starting at $\left(X_{v_{\alpha}}(t), t\right)$ actually passes through the origin, but this is false in general. In fact, after time $t$, most $\alpha$-rays will have coalesced with other rays, and the crossings with the $x$-axis will be quite far apart; we would conjecture they are order $t^{2 / 3}$ apart.

Proposition 7.3 allows us to use Theorem 2.1 and Lemma 5.2 to prove a strong law for the second class particle in the case of $v_{\alpha}$. However, we are able to prove a strong law even for deterministic initial conditions that satisfy a density property given in the following lemma, whose proof is very similar to Lemma 5.2 and can be found in Sect. 9:

Lemma 7.4 Suppose $v \in \mathcal{N}$. Assume that

$$
\liminf _{d \rightarrow \infty} \inf _{z \in[-4 d, 4 d]} \frac{v((z-d, z+d])}{2 d} \geq \frac{\gamma}{2} \sqrt{\tan \alpha} .
$$

Let $\varepsilon>0$. Then, with probability one,

$$
\liminf _{t \rightarrow \infty} \frac{Z_{v}((1+\varepsilon) t,(\tan \alpha) t)}{t}>0 .
$$

Now assume that

$$
\limsup _{d \rightarrow \infty} \sup _{z \in[-4 d, 4 d]} \frac{v((z-d, z+d])}{2 d} \leq \frac{\gamma}{2} \sqrt{\tan \alpha .}
$$

Then, with probability one,

$$
\limsup _{t \rightarrow \infty} \frac{Z_{v}((1-\varepsilon) t,(\tan \alpha) t)}{t}<0 .
$$

Note that in our density condition, we do not allow the midpoint of the interval to be much larger than $d$. The reason for this might be more clear if we think of a Poisson process: if we fix $d$, we can always find some $z \in \mathbb{R}$ such that the interval $[z-d, z+d]$ is empty! With this Lemma we can proof the following result: 
Theorem 7.5 Assume that

$$
\lim _{x \rightarrow \infty} \frac{v(x)}{x}=\lim _{x \rightarrow-\infty} \frac{v(x)}{x}=\frac{\gamma}{2} \sqrt{\tan \alpha}
$$

Then, with probability one,

$$
\lim _{t \rightarrow \infty} \frac{X_{v}(t)}{t}=\frac{1}{\tan \alpha}
$$

Proof It is enough to prove the statement for $\alpha=5 \pi / 4$. Suppose $\varepsilon>0$ and $X_{\nu}(t) \leq$ $t-2 \varepsilon t$. Define $n=\lfloor t\rfloor$. Then for $t$ large enough, we have

$$
X_{v}(n) \leq(1-2 \varepsilon) n+1+2 \varepsilon \leq(1-\varepsilon) n .
$$

By (7.2), this implies that $Z_{v}((1-\varepsilon) n, n) \geq 0$. If we can show that $v$ satisfies (7.4), then we can use Lemma 7.4 to see that $Z_{v}((1-\varepsilon) n, n) \geq 0$ can happen only for finitely many $n \geq 1$, which gives

$$
\liminf _{t \rightarrow \infty} \frac{X_{v}(t)}{t} \geq 1
$$

Bounding the limit from above can be done using the analogous argument. To see that (7.4) indeed holds, remark that for all $1>\eta>0$, there exists $R>1$ such that for all $z \geq R$,

$$
\left|\frac{\nu((0, z])}{z}-\frac{1}{2} \gamma\right|<\eta \text { and }\left|\frac{\nu((-z, 0])}{z}-\frac{1}{2} \gamma\right|<\eta \text {. }
$$

Choose $M>1$ such that $v([-R, R])<M$. Now choose $d>M R / \eta$. If $z>d+R$ or $z<-d-R$, we get that

$$
v((z-d, z+d]) \leq d \gamma+(2|z|+2 d) \eta \leq d \gamma+10 d \eta
$$

If $|z+d|<R$

$$
v((z-d, z+d]) \leq \frac{1}{2}(2 d+R) \gamma+M+|z-d| \eta \leq d \gamma+\left(5 d+2+\frac{1}{2} \gamma\right) \eta,
$$

and a similar bound holds when $|z-d|<R$. This proves that $v$ satisfies (7.4).

\section{Lattice last-passage percolation}

In the lattice last-passage percolation model we have a collection $\left\{\omega_{\mathbf{z}}: \mathbf{z} \in \mathbb{Z}^{2}\right\}$ of i.i.d. non-negative random variables indexed by lattice points $\mathbf{z} \in \mathbb{Z}^{2}$. In this set-up, 
one can define last-passage times for $\mathbf{x} \leq \mathbf{y}$ (we put an $\ell$ on the top to indicate that it refers to the lattice model) by maximizing over up-right paths connecting $\mathbf{x}$ to $\mathbf{y}$ :

$$
L^{\ell}(\mathbf{x}, \mathbf{y}):=\max _{\varpi \in \Pi(\mathbf{x}, \mathbf{y})}\left\{\sum_{\mathbf{x}^{\prime} \in \varpi} \omega_{\mathbf{x}^{\prime}}\right\} .
$$

Similarly to the previous case, one can start with a non-decreasing process $v^{\ell}=$ $\left(v^{\ell}(z): z \in \mathbb{Z}\right)$ and define the evolution by

$$
M_{t}^{v^{\ell}}((x, y]):=L_{v^{\ell}}(y, t)-L_{v^{\ell}}(x, t), \quad(x, y \in \mathbb{Z}, t \geq 0)
$$

where

$$
L_{v^{\ell}}(x, t):=\sup _{z \leq x}\left\{v^{\ell}(z)+L^{\ell}((z, 0),(x, t))\right\}
$$

In this set-up, $v^{\ell}$ must have a left density analogous to (4.3), i.e.:

$$
\liminf _{z \rightarrow-\infty} \frac{v^{\ell}(z)}{z}>1
$$

For a survey in lattice last-passage percolation (and its connection with the totally asymmetric exclusion process) we address to [15].

If $\omega_{\mathbf{z}}$ has an exponential distribution of parameter one, then the limit shape is given by $(\sqrt{x}+\sqrt{t})^{2}$ and a result similar to Theorem 2.2 holds [10]. This allows us to construct Busemann functions $B^{\ell}$ for $\alpha \in(\pi, 3 \pi / 2)$. The same method developed to prove Theorem 5.3 can be applied to this case. Since for exponential weights we also know the invariant measure, we have that:

Theorem 8.1 For $\alpha \in(\pi, 3 \pi / 2)$ let

$$
v_{\alpha}^{\ell}(x)=B_{\alpha}^{\ell}((0,0),(x, 0)) \text { for } x \in \mathbb{Z}
$$

Then $v_{\alpha}^{\ell}$ is the unique ergodic process on $\mathbb{Z}$ that satisfies

$$
M_{t}^{v_{\alpha}^{\ell}} \stackrel{\mathcal{D}}{=} v_{\alpha}^{\ell} \text { for all } t \geq 0
$$

In particular, for any $x, y \in \mathbb{Z}, M_{t}^{v_{\alpha}^{\ell}}(x, y)$ is distributed like a sum of i.i.d. exponential random variables of intensity

$$
\rho(\alpha):=\frac{1}{1+\sqrt{\tan \alpha}} .
$$


We remark that $\rho(\alpha)$ is obtained by solving

$$
(1+\sqrt{\tan \alpha})^{2}=\frac{1}{\rho}+\frac{\tan \alpha}{1-\rho} .
$$

The left-hand side corresponds to the limit shape of the lattice model, in direction $(1, \tan \alpha)$, while the right-hand side corresponds to summing up, in the same direction, the expectation of the equilibrium process in the horizontal and vertical axis. (Compare this with (5.6) and its proof.) The dual process on the vertical axis, denoted by $v_{\alpha}^{\ell *}$, is distributed like a sum of i.i.d. exponential weights of intensity $1-\rho(\alpha)$ (see also [3]).

To develop our method in the model with general i.i.d. weights, one would need an inequality similar to (9.2) for the respective limit shape. This is, however, one of the most challenging problems in lattice last (and first) passage percolation models.

Acknowledgments Part of this work was done during our stay at the Institute Henri Poincare, Centre Emile Borel, attending the program Interacting Particle Systems, Statistical Mechanics and Probability Theory (September 5-December 19, 2008). Both authors wish to thank the organizers and the Institute for their hospitality and support during our stay there. We would also like to thank an anonymous referee for many helpful suggestions to improve this manuscript.

Open Access This article is distributed under the terms of the Creative Commons Attribution Noncommercial License which permits any noncommercial use, distribution, and reproduction in any medium, provided the original author(s) and source are credited.

\section{Appendix}

\subsection{Proof of Proposition 3.1}

The translation invariance of the underlying compound two-dimensional Poisson process,

$$
\left\{\omega_{\mathbf{q}}: \mathbf{q} \in(\mathbf{P}+\mathbf{z})\right\} \stackrel{\mathcal{D}}{=}\left\{\omega_{\mathbf{p}}: \mathbf{p} \in \mathbf{P}\right\}
$$

implies (1).

Anti-symmetry follows directly from the definition of the Busemann function. Now, by taking a coalescence point $\mathbf{c}$ between $\varpi_{\alpha}(\mathbf{x}), \varpi_{\alpha}(\mathbf{y})$ and $\varpi_{\alpha}(\mathbf{z})$ we have that

$$
\begin{aligned}
B_{\alpha}(\mathbf{x}, \mathbf{z}) & =L(\mathbf{c}, \mathbf{z})-L(\mathbf{c}, \mathbf{x}) \\
& =L(\mathbf{c}, \mathbf{z})-L(\mathbf{c}, \mathbf{y})+L(\mathbf{c}, \mathbf{y})-L(\mathbf{c}, \mathbf{x}) \\
& =B_{\alpha}(\mathbf{y}, \mathbf{z})+B_{\alpha}(\mathbf{x}, \mathbf{y}),
\end{aligned}
$$

which clearly shows additivity, and finishes the proof of (2).

Define $S$ as the reflection in the diagonal $x=t$. Then $S(\mathbf{P})$ has the same distribution as $\mathbf{P}$, and $\mathbf{x} \leq \mathbf{y} \Leftrightarrow S(\mathbf{x}) \leq S(\mathbf{y})$. This shows that for all $\mathbf{x}, \mathbf{y} \in \mathbb{R}^{2}$,

$$
L(\mathbf{x}, \mathbf{y}) \stackrel{\mathcal{D}}{=} L(S(\mathbf{x}), S(\mathbf{y})) .
$$


Note that the lowest geodesic in the reflected case is not necessarily the reflection of the original lowest geodesic. However, we can now use (3.2) to prove (3).

Since $L(\mathbf{c}, \mathbf{y}) \geq L(\mathbf{c}, \mathbf{x})$ whenever $\mathbf{c} \leq \mathbf{x} \leq \mathbf{y}$ we have that $B_{\alpha}(\mathbf{x}, \mathbf{y}) \geq 0$ whenever $\mathbf{x} \leq \mathbf{y}$. Note that

$$
B_{\alpha}(\mathbf{x}, \mathbf{x}+(1,1)) \geq L(\mathbf{x}, \mathbf{x}+(1,1)) .
$$

Clearly, $\mathbb{E} L(\mathbf{x}, \mathbf{x}+(1,1))>0$ (there is a positive probability that a Poisson point will fall in between $\mathbf{x}$ and $\mathbf{x}+(1,1))$. Also,

$$
\mathbb{E} B_{\alpha}(\mathbf{x}, \mathbf{x}+(1,1))=\mathbb{E} B_{\alpha}(\mathbf{x}, \mathbf{x}+(1,0))+\mathbb{E} B_{\alpha}(\mathbf{x}, \mathbf{x}+(0,1)) .
$$

Property (3) then shows that

$$
\mathbb{E} B_{\alpha}(\mathbf{x}, \mathbf{x}+(1,0))=\mathbb{E} B_{\alpha}(\mathbf{x}, \mathbf{x}+(0,1))>0 .
$$

Properties (1) and (2) show that for $h>0, \mathbb{E} B_{\alpha}(\mathbf{x},(h, 0))=h \mathbb{E} B_{\alpha}(\mathbf{x}, \mathbf{x}+(1,0))>0$, and the same for the vertical direction.

To prove that its expected value is finite, without loss of generality, assume that $\mathbf{y}=0$, that $\alpha=5 \pi / 4$, and that $\mathbf{x}=(-1,-1)$. Let $\mathbf{z}$ be the intersection point between $\varpi_{5 \pi / 4}(\mathbf{0})$ and the one-dimensional boundary of $\{\mathbf{p}: \mathbf{p} \leq \mathbf{x}\}$. By taking a coalescence point $\mathbf{c} \leq \mathbf{z} \leq \mathbf{x}$, we have that

$$
\begin{aligned}
B_{5 \pi / 4}(\mathbf{x}, \mathbf{0}) & =L(\mathbf{c}, \mathbf{0})-L(\mathbf{c}, \mathbf{x}) \\
& =L(\mathbf{c}, \mathbf{z})+L(\mathbf{z}, \mathbf{0})-L(\mathbf{c}, \mathbf{x}) \\
& =L(\mathbf{z}, \mathbf{0})-\{L(\mathbf{c}, \mathbf{x})-L(\mathbf{c}, \mathbf{z})\} \\
& \leq L(\mathbf{z}, \mathbf{0}) .
\end{aligned}
$$

Now assume that $c_{0}>0$ is a small constant and that $|\mathbf{z}| \leq c_{0} r$. Then

$$
L(\mathbf{z}, \mathbf{0}) \leq \min \left\{L\left(\left(-1,-c_{0} r\right), \mathbf{0}\right), L\left(\left(-c_{0} r,-1\right), \mathbf{0}\right)\right\} \leq P\left(c_{0} r\right)
$$

where $P(r)$ denotes the number of Poisson points in the set $[-1,0] \times\left[-c_{0} r, 0\right] \cup$ $\left[-c_{0} r, 0\right] \times[-1,0]$. Hence

$$
\mathbb{P}(L(\mathbf{z}, \mathbf{0}) \geq r) \leq \mathbb{P}\left(|\mathbf{z}|>c_{0} r\right)+\mathbb{P}\left(P\left(c_{0} r\right) \geq r\right) .
$$

By choosing $c_{0}$ small enough, one can make $\mathbb{P}\left(P\left(c_{0} r\right) \geq r\right)$ integrable over $r>0$. It is therefore enough to prove that $\mathbb{P}\left(|\mathbf{z}|>c_{0} r\right)$ is integrable over $r>0$. We first consider the case where $\mathbf{z} \leq\left(-1,-c_{0} r\right)$; the case $\mathbf{z} \leq\left(-c_{0} r,-1\right)$ can be handled in exactly the same way. For this we need some notation: denote by $\operatorname{Co}(\mathbf{p}, \theta)$ the cone starting at $\mathbf{0}$, with symmetry axis the line through $\mathbf{0}$ and $\mathbf{p}$, and with $\theta$ equal to half the top-angle. Furthermore, define for $\mathbf{p} \leq \mathbf{0}$,

$$
R_{\mathbf{0}}^{\text {out }}(\mathbf{p})=\{\mathbf{q} \leq \mathbf{p}: \mathbf{p} \in \varpi(\mathbf{q}, \mathbf{0})\}
$$


By Lemma 2.3 of [7] ( $\delta$-straightness of geodesics): fix $\delta \in(0,1 / 4)$ and $\theta \in(0, \pi / 4)$. There exist $c_{1}, c_{2}, \kappa, M>0$ such that for all $\mathbf{p} \in \operatorname{Co}((1,1), \theta)$ with $|\mathbf{p}|>M$, we have

$$
\mathbb{P}\left(\left(\bigcup_{\mathbf{p}^{\prime} \in \mathbf{p}+[0,1]^{2}} R_{\mathbf{0}}^{\text {out }}\left(\mathbf{p}^{\prime}\right)\right) \subset \operatorname{Co}\left(\mathbf{p},|\mathbf{p}|^{-\delta}\right)\right) \geq 1-c_{1} e^{-c_{2}|\mathbf{p}|^{\kappa}}
$$

Let $b:=(\tan (23 \pi / 16))^{-1}, b^{\prime}:=(\tan (21 \pi / 16))^{-1}$ and $\mathbf{p}_{n}:=(-b n,-n)$. Fix $n_{0}>0$ big enough so that $\operatorname{Co}\left(\mathbf{p}_{n},\left|\mathbf{p}_{n}\right|^{-\delta}\right)$ does not intersect $\left\{\left(-b^{\prime} t,-t\right): t>0\right\}$ for all $n \geq n_{0}$ (recall that $\delta \in(0,1 / 4)$ is fixed). Since the asymptotic direction of $\varpi_{5 \pi / 4}(\mathbf{0})$ equals $(-1,-1)$ it will eventually be to left of $\left\{\left(-b^{\prime} t,-t\right): t>0\right\}$. Therefore, if $\mathbf{z} \leq$ $\left(-1,-c_{0} r\right)$ and $c_{0} r \geq n_{0}$, there must be an $n \geq c_{0} r$ and $\mathbf{p}_{n}^{\prime} \in\left(\mathbf{p}_{n}+[0,1]^{2}\right) \cap \varpi_{5 \pi / 4}(\mathbf{0})$ such that

$$
R_{\mathbf{0}}^{\text {out }}\left(\mathbf{p}_{n}^{\prime}\right) \not \subset \operatorname{Co}\left(\mathbf{p}_{n},\left|\mathbf{p}_{n}\right|^{-\delta}\right)
$$

Using (9.1), we see that

$$
\mathbb{P}\left(\mathbf{z} \leq\left(-1,-c_{0} r\right)\right) \leq \sum_{n \geq c_{0} r} c_{1} e^{-c_{2}\left|\mathbf{p}_{n}\right|^{\kappa}},
$$

for $c_{0} r>\max \left\{n_{0}, M\right\}$, which shows integrability. This finally implies that $\mathbb{E} L(\mathbf{z}, \mathbf{0})<$ $\infty$, and consequently, $\mathbb{E} B_{5 \pi / 4}(\mathbf{x}, \mathbf{0})<\infty$.

By additivity and anti-symmetry, to prove cadlag, we can restrict our attention to $\mathbf{y}=(0,0), \lambda=0$ and $\mathbf{q}=(1,0)$ so that $\epsilon$ is varying on the horizontal direction close to the origin. For different values of $\mathbf{y}$ the argument is similar. Choose $\mathbf{c}$ as the coalescing point of $\varpi_{\alpha}(\mathbf{0})$ and $\varpi_{\alpha}(\mathbf{q})$. Furthermore, define $y<0$ such that $(0, y)$ is the crossing point of $\varpi_{\alpha}(\mathbf{q})$ with the $y$-axis. Then for every $\lambda \in[0,1]$, we have that

$$
B_{\alpha}(\mathbf{0}, \lambda \mathbf{q})=L(\mathbf{c}, \lambda \mathbf{q})-L(\mathbf{c}, \mathbf{0})
$$

This is because the $\alpha$-rays starting at $\lambda \mathbf{q}$ are wedged in between $\varpi_{\alpha}(\mathbf{0})$ and $\varpi_{\alpha}(\mathbf{q})$. Furthermore, for $\lambda$ small enough, there will be no Poisson points in the rectangle $[0, \lambda] \times[y, 0]$, which means that for those $\lambda, L(\mathbf{c}, \lambda \mathbf{q})=L(\mathbf{c}, \mathbf{0})$, and therefore

$$
B_{\alpha}(\mathbf{0}, \lambda \mathbf{q})=0
$$

This proves right continuity. The existence of the left limit follows from monotonicity.

9.2 Lemma from real analysis

Lemma 9.1 Let $I=[a, b]$ be a compact interval. Suppose $f: I \rightarrow \mathbb{R}$ is a non-decreasing right-continuous function and $g: I \rightarrow \mathbb{R}$ is a non-increasing 
left-continuous function. Then the function $h=f+g$ attains its maximum on I, and the set of maxima is closed.

Proof Clearly, for every $x \in I, h(x) \leq f(b)+g(a)$, so $M:=\sup _{x \in I} h(x)<+\infty$. Since $I$ is compact, we can choose a converging sequence $x_{n}$ such that $h\left(x_{n}\right) \rightarrow M$. Denote $x_{0}=\lim _{n \rightarrow \infty} x_{n} \in I$. Now we can choose a subsequence $x_{k(n)}$ such that either $x_{k(n)} \uparrow x_{0}$ or $x_{k(n)} \downarrow x_{0}$. In the first case, $f\left(x_{0}\right) \geq f\left(x_{k(n)}\right)$ and $g\left(x_{0}\right)=$ $\lim _{n \rightarrow \infty} g\left(x_{k(n)}\right)$, so $h\left(x_{0}\right)=M$ (note that we always have that $h\left(x_{0}\right) \leq M$ ). In the second case, $g\left(x_{0}\right) \geq g\left(x_{k(n)}\right)$ and $f\left(x_{0}\right)=\lim _{n \rightarrow \infty} f\left(x_{k(n)}\right)$, so we also have that $h\left(x_{0}\right)=M$. This proves that the maximum is attained. The fact that the set of maxima is closed follows in the same way by choosing a converging sequence $x_{n}$ with $h\left(x_{n}\right)=M$.

\subsection{Proof of Lemmas 5.2 and 7.4}

Proof of Lemma 5.2 We start with the proof for $Z_{v}$. Using the transformation (2.4), we can assume, without loss of generality, that $\alpha=5 \pi / 4$. The proof of this lemma is based on the following elementary estimate for the shape function $f(x, t)=\gamma \sqrt{x t}$ : for $s \geq 0$

$$
f(t+s, t)-f(t, t) \leq\left\{\begin{array}{cl}
\frac{\gamma}{2} s-\frac{\gamma}{32} \frac{s^{2}}{t} & \text { if } s \leq 8 t \\
\frac{\gamma}{\sqrt{8}} s & \text { if } s \geq 8 t
\end{array}\right.
$$

Fix $\varepsilon>0$ and suppose that $Z_{v, h}(t):=Z_{v}(t+h, t) \leq-\varepsilon t-1$. Define $k=-\left\lceil Z_{v}(t)\right\rceil$ and $n=\lfloor t+h\rfloor$. It follows that

$$
v(-k+1)+L((-k, 0),(n+1, n+1))-L((0,0),(n, n)) \geq 0 .
$$

Choose $\eta>0$ small enough (we will see how small). For $t$ big enough, we know from (5.4) that

$$
v(-k+1) \leq-\frac{1}{2} \gamma k+\eta k
$$

Here we use that $k \geq \varepsilon t$. This implies that for $t$ big enough,

$$
L((-k, 0),(n+1, n+1))-L((0,0),(n, n)) \geq \frac{1}{2} \gamma k-\eta k .
$$

A straightforward application of Theorem 2.1 and Borel-Cantelli shows that for $t$ big enough (and therefore for $n$ big enough), we will have that

$$
|L((0,0),(n, n))-f(n, n)| \leq \eta n .
$$


Below we will work out a more complicated application of Theorem 2.1. We now have for $t$ big enough

$$
L((-k, 0),(n+1, n+1))-f(n, n) \geq \frac{1}{2} \gamma k-\eta k-\eta n .
$$

For $t$ big enough, we will have that $k \geq \frac{1}{2} \varepsilon n$ (since $h$ is fixed). Now we subtract $f(n+k, n)-f(n, n)$ from both sides of the inequality and use (9.2):

$$
\begin{aligned}
L((-k, 0),(n+1, n+1))-f(n+k, n) & \geq\left\{\begin{array}{l}
\frac{\gamma}{32} \frac{k^{2}}{n}-\eta k-\eta n \text { if } \frac{1}{2} \varepsilon n \leq k \leq 8 n, \\
\frac{\gamma}{\sqrt{8}} k-\eta k-\eta n \text { if } k \geq 8 n
\end{array}\right. \\
& \geq\left\{\begin{array}{l}
\frac{\varepsilon^{2} \gamma}{128} n-9 \eta n \text { if } k \leq 8 n, \\
\frac{\gamma}{\sqrt{8}} k-2 \eta k \text { if } k \geq 8 n .
\end{array}\right.
\end{aligned}
$$

Therefore, if the set $\left\{t \geq 0: Z_{v}(t, t) \leq-\varepsilon t-1\right\}$ is unbounded, it follows that for some small $\eta>0$, the events

$$
\left\{|L((-k, 0),(n+1, n+1))-f(n+k, n)|>\left\{\begin{array}{ll}
\eta n & \text { if } k \leq 8 n \\
\eta k & \text { if } k \geq 8 n .
\end{array}\right\}\right.
$$

happen infinitely often for $n \geq 1$ and $k \geq 1$. Using Borel-Cantelli, this will have zero probability if for all $\eta>0$,

$$
\sum_{n=1}^{\infty} \sum_{k=0}^{8 n} \mathbb{P}(|L((-k, 0),(n+1, n+1))-f(n+k, n)|>\eta n)<+\infty
$$

and

$$
\sum_{n=1}^{\infty} \sum_{k=8 n}^{\infty} \mathbb{P}(|L((-k, 0),(n+1, n+1))-f(n+k, n)|>\eta k)<+\infty
$$

Theorem 2.1 gives us some control on the fluctuations of $L$ about its asymptotic shape. Note that for $n$ big enough, and $0 \leq k \leq 8 n$, we have

$$
\begin{aligned}
& \mathbb{P}(|L((-k, 0),(n+1, n+1))-f(n+k, n)|>\eta n) \\
& \quad=\mathbb{P}(|L(\mathbf{0}, \sqrt{(n+1+k)(n+1)}(1,1))-f(n+k, n)|>\eta n) .
\end{aligned}
$$

Define $r=\sqrt{(n+1+k)(n+1)}$ and $u=n^{2 / 3}$. If we choose $n$ large enough, we can make sure that $u<\eta n / 2$ and

$$
|f(n+k, n)-\gamma r|<\eta n / 2 .
$$


Also, for $n$ large enough, we have that $u \in\left[c_{3} \sqrt{r} \log ^{2} r, c_{4} r^{3 / 2} \log r\right]$ (see Theorem 2.1). This implies, using Theorem 2.1, that there exist $c_{1}, c_{2}>0$ such that for $n$ large enough and $0 \leq k \leq 8 n$,

$$
\begin{aligned}
\mathbb{P}(|L((-k, 0),(n+1, n+1))-f(n+k, n)|>\eta n) & \leq \mathbb{P}(|L(\mathbf{0},(r, r))-\gamma r|>u) \\
& \leq c_{1} \exp \left(-c_{2} n^{1 / 6} / \log (n)\right)
\end{aligned}
$$

This clearly proves (9.4). For $k \geq 8 n$, we define $u=k^{2 / 3}$, and in a similar way we find that

$$
\mathbb{P}(|L((-k, 0),(n+1, n+1))-f(n+k, n)|>\eta k) \leq c_{1} \exp \left(-c_{2} k^{1 / 6} / \log (k)\right) .
$$

This proves (9.5).

The proof that the set $\left\{t \geq 0: Z_{v}(t+h, t) \geq \varepsilon t+1\right\}$ is bounded with probability 1 is actually easier, because we will have that $0 \leq k \leq n$. Therefore, we only need to use the following bound on the shape function $f(x, t)=\gamma \sqrt{x t}$ : for $0 \leq s \leq t$

$$
f(t-s, t) \leq \frac{1}{2} \gamma s-\frac{\gamma}{8} \frac{s^{2}}{t} .
$$

The remainder of the argument is similar to the previous case.

Now we consider $\tilde{Z}_{v, h}$. Since for all $t$ we have $\tilde{Z}_{v, h}(t, t) \stackrel{\mathcal{D}}{=} Z_{v}(t+h, t)$ (but not as processes!), the Borel-Cantelli type arguments for $Z_{v, h}$ hold in this case as well, mutatis mutandis.

Proof of Lemma 7.4 The proof of this Lemma relies on the proof of Lemma 5.2. As usual, we will assume without loss of generality that $\alpha=5 \pi / 4$. We will start with the second statement. We need to prove that for all $\eta>0$ small enough, the set $\left\{t \geq 0: Z_{v}((1+2 \varepsilon) t, t) \leq \eta t\right\}$ is bounded with probability one. Choose $\eta<\varepsilon / 2$. Define the translated measure $v_{2 \varepsilon t}$ such that for all $b \geq a$,

$$
\nu_{2 \varepsilon t}((a, b])=v((a+2 \varepsilon t, b+2 \varepsilon t]) .
$$

Using Definition 4.1, we see that for $z \in \mathbb{R}$,

$$
v_{2 \varepsilon t}(z)=v(z+2 \varepsilon t)-v(2 \varepsilon t)
$$

Define $k=-\left\lceil Z_{v}((1+2 \varepsilon) t, t)-2 \varepsilon t\right\rceil$ and $n=\lfloor t\rfloor$. If $t$ is such that $Z_{v}((1+2 \varepsilon) t, t) \leq$ $\eta t$, then

$$
\begin{gathered}
v(-k+1+2 \varepsilon t)+L((-k+2 \varepsilon t, 0),((1+2 \varepsilon) t, t)) \\
-[v(2 \varepsilon t)+L((2 \varepsilon t, 0),((1+2 \varepsilon) t, t))] \geq 0 .
\end{gathered}
$$

This event has the same probability as the event

$$
v_{2 \varepsilon t}(-k+1)+L((-k, 0),(t, t))-L((0,0),(t, t)) \geq 0 .
$$


We know that $k \geq \varepsilon t$ (since $\eta<\varepsilon$ ). We can use (7.4) to see that for $t$ big enough,

$$
-v_{2 \varepsilon t}(-k+1)=v((-k+1+2 \varepsilon t, 2 \varepsilon t]) \geq \frac{1}{2} \gamma k-\eta k .
$$

To see this, define half the length of the interval by $d=(k-1) / 2$, and the midpoint by $z=\varepsilon t-(k-1) / 2$. We see then see that

$$
|z| \leq 4 d
$$

This gives us, using the fact that $n \leq t \leq n+1$,

$$
L((-k, 0),(n+1, n+1))-L((0,0),(n, n)) \geq \frac{1}{2} \gamma k-\eta k .
$$

This is exactly Equation (9.3) in the proof of Lemma 5.2, and we can follow that proof from this point. The first statement of the Lemma follows similarly.

\section{References}

1. Aldous, D., Diaconis, P.: Hammersley's interacting particle system and longest increasing subsequences. Probab. Theory Relat. Fields 103, 199-213 (1995)

2. Baik, J., Rains, E.: Limiting distributions for a polynuclear growth model with external sources. J. Stat. Phys. 102, 1085-1132 (2001)

3. Balázs, M., Cator, E.A., Seppäläinen, T.: Cube root fluctuations for the corner growth model associated to the exclusion process. Electron. J. Probab. 11, 1094-1132 (2006)

4. Busemann, H.: The Geometry of Geodesics. Academic Press, New York (1955)

5. Cator, E.A., Groeneboom, P.: Hammersley's process with sources and sinks. Ann. Probab. 33, 879903 (2005)

6. Cator, E.A., Groeneboom, P.: Second class particles and cube root asymptotics for Hammersley's process. Ann. Probab. 34, 1273-1295 (2006)

7. Cator, E.A., Pimentel, L.P.R.: A shape theorem and semi-infinite geodesics for the Hammersley model with random weights. ALEA (2010). ArXiv:1001.4706 (to appear)

8. Ferrari, P.A., Martin, J.B.: Multiclass Hammersley-Aldous-Diaconis process and multiclass-customer queues. Ann. Inst. H. Poincare 99, 305-319 (2007, to appear)

9. Ferrari, P.A., Martin, J.B., Pimentel, L.P.R.: A phase transition for competition interfaces. Ann. Appl. Probab. 19, 281-317 (2009)

10. Ferrari, P.A., Pimentel, L.P.R.: Competition interfaces and second class particles. Ann. Probab. 33, 1235-1254 (2005)

11. Hammersley, J.M.: A few seedlings of research. In: Proceedings of the Sixth Berkeley Symposium on Mathematical Statistics and Probabability, vol. 1, pp. 345-394. University of California Press, CA (1972)

12. Howard, C.D., Newman, C.M.: Geodesics and spanning trees for euclidean first passage percolation. Ann. Probab. 29, 577-623 (2001)

13. Kesten, H.: On the speed of convergence in first passage percolation. Ann. Appl. Probab. 3, 296-338 (1993)

14. Newman, C.M.: A surface view of first-passage percolation. In: Proceedings of the International Congress of Mathematicians, vols. 1, 2, pp. 1017-1023. Birkhäuser, Basel (1995) (Zürich, 1994)

15. Seppäläien, T.: Directed random growth models on the plane. In: Analysis and stochastics of growth processes and interfaces models, pp. 9-38. Oxford University press, NY (2009)

16. Wüthrich, M.: Asymptotic behavior of semi-infinite geodesics for maximal increasing subsequences in the plane. In: Sidoravicius, V. (ed.) In and Out of Equilibrium, pp. 205-226. Birkhäuser, Basel (2002) 